\title{
Perturbation of $n$ dimensional AQ - mixed type functional equation via Banach spaces and Banach algebra : Hyers direct and alternative fixed point methods
}

\author{
M. Arunkumar \\ Department of Mathematics, Government Arts College, Tiruvannamalai - 606 603, TamilNadu, India \\ Email: annarun2002@yahoo.co.in
}

Copyright (C)2014 M. Arunkumar. This is an open access article distributed under the Creative Commons Attribution License, which permits unrestricted use, distribution, and reproduction in any medium, provided the original work is properly cited.

\begin{abstract}
In this paper, the authors obtain the general solution and generalized Ulam - Hyers stability of $n$ dimensional additive quadratic functional equation

$\sum_{i=0}^{n}\left[f\left(x_{2 i}+x_{2 i+1}\right)+f\left(x_{2 i}-x_{2 i+1}\right)\right]=\sum_{i=0}^{n}\left[2 f\left(x_{2 i}\right)+f\left(x_{2 i+1}\right)+f\left(-x_{2 i+1}\right)\right]$

in Banach spaces using direct and fixed point methods. We also investigate the stability of the above equation in Banach algebra using direct and fixed point approach.

Keywords: Additive functional equations, Mixed type AQ functional equation, quadratic functional equation, Ulam - Hyers stability.
\end{abstract}

\section{Introduction}

The study of stability problems for functional equations is related to a question of Ulam [28] concerning the stability of group homomorphisms was affirmatively answered for Banach spaces by Hyers [13]. Subsequently, this result of Hyers was generalized by Aoki [2] for additive mappings and by Rassias [23] for linear mappings by considering an unbounded Cauchy difference.

The article of Rassias [23] has provided a lot of influence in the development of what we now call generalized Ulam-Hyers stability of functional equations. The terminology generalized Ulam - Hyers stability originates from these historical backgrounds. These terminologies are also applied to the case of other functional equations. For more detailed definitions of such terminologies, one can refer to $[9,14,17,19]$.

Over the last seven decades, the above Ulam problem was tackled by numerous authors who provided solutions in various forms of functional equations like additive, quadratic, cubic, quartic, mixed type functional equations involving only these types of functional equations were discussed. We refer the interested readers for more information on such problems to the monographs $[1,9,12,14,17,19]$. 
One of the most famous functional equations is the additive functional equation

$f(x+y)=f(x)+f(y)$.

In 1821, it was first solved by A.L. Cauchy in the class of continuous real-valued functions. It is often called an additive Cauchy functional equation in honor of Cauchy (see [19]). The theory of additive functional equations is frequently applied to the development of theories of other functional equations. Moreover, the properties of additive functional equations are powerful tools in almost every field of natural and social sciences. The additive function $f(x)=c x$ is the solution of the additive functional equation (1). The stability of the above functional equation was excellently discussed in $[2,10,13,21,23,26]$.

The quadratic function $f(x)=c x^{2}$ satisfies the functional equation

$f(x+y)+f(x-y)=2 f(x)+2 f(y)$,

and therefore the equation (2) is called quadratic functional equation.

The Hyers - Ulam stability theorem for the quadratic functional equation (2) was proved by F.Skof [27] for the functions $f: E_{1} \rightarrow E_{2}$ where $E_{1}$ is a normed space and $E_{2}$ be a Banach space. The result of Skof is still true if the relevant domain $E_{1}$ is replaced by an Abelian group and it was delt by P.W.Cholewa [7]. S.Czerwik [8] proved the Hyers-Ulam-Rassias stability of the quadratic functional equation (2). This result further generalized by Th.M.Rassais [25], C.Borelli, and G.L.Forti [5].

In 2006, K.W. Jun and H.M. Kim [15] introduced the following generalized additive and quadratic type functional equation

$f\left(\sum_{i=1}^{n} x_{i}\right)+(n-2) \sum_{i=1}^{n} f\left(x_{i}\right)=\sum_{1 \leq i<j \leq n} f\left(x_{i}+x_{j}\right)$

in the class of function between real vector spaces. For $n=3$, Pl.Kannappan proved that a function $f$ satisfies the functional equation (3) if and only if there exists a symmetric bi-additive function $A$ and additive function $B$ such that $f(x)=B(x, x)+A(x)$ for all $x$ (see [18]). The Hyers-Ulam stability for the equation (3) when $n=3$ was proved by S.M. Jung [16]. The Hyers-Ulam-Rassias stability for the equation (3) when $n=4$ was also investigated by I.S. Chang et al., [6].

Recently, M. Arunkumar and S. Karthikeyan [3] introduced and established the general solution and generalized Ulam-Hyers stability of $n$-dimensional mixed type additive and quadratic functional equation of the form

$$
\begin{aligned}
f\left(-x_{1}\right)+f\left(2 x_{1}-\sum_{i=2}^{n} x_{i}\right) & +f\left(2 \sum_{i=2}^{n} x_{i}\right)+f\left(x_{1}+\sum_{i=2}^{n} x_{i}\right)-f\left(-x_{1}-\sum_{i=2}^{n} x_{i}\right) \\
& -f\left(x_{1}-\sum_{i=2}^{n} x_{i}\right)-f\left(-x_{1}+\sum_{i=2}^{n} x_{i}\right)=3 f\left(x_{1}\right)+3 f\left(\sum_{i=2}^{n} x_{i}\right)
\end{aligned}
$$

in Banach spaces.

Very recently, M.J. Rassias et. al., [24] introduced the Leibniz type additive-quadratic functional equation of the form

$f(x-t)+f(y-t)+f(z-t)=3 f\left(\frac{x+y+z}{3}-t\right)+f\left(\frac{2 x-y-z}{3}\right)+f\left(\frac{-x+2 y-z}{3}\right)+f\left(\frac{-x-y+2 z}{3}\right)$

and obtained its general solution and generalized Ulam - Hyers stability of Leibniz AQ - mixed type functional equation in Quasi-Beta normed space using direct and fixed point methods.

In this paper, the authors obtain the general solution and generalized Ulam - Hyers stability of $n$ dimensional additive quadratic functional equation

$\sum_{i=0}^{n}\left[f\left(x_{2 i}+x_{2 i+1}\right)+f\left(x_{2 i}-x_{2 i+1}\right)\right]=\sum_{i=0}^{n}\left[2 f\left(x_{2 i}\right)+f\left(x_{2 i+1}\right)+f\left(-x_{2 i+1}\right)\right]$

in Banach spaces using direct and fixed point methods. We also investigate the stability of the above equation in Banach algebra derivations using direct and fixed point approach. 


\section{General solution}

In this section, the general solution of the functional equation (6) is given. Through out this section let as assume $E$ and $F$ be real vector spaces.

Lemma 2.1 If an odd mapping $f: E \rightarrow F$ satisfies the functional equation (1) if and only if $f: E \rightarrow F$ satisfies the functional equation (6).

Proof. Assume $f: E \rightarrow F$ satisfies the functional equation (1). Letting $x=y=0$ in (1), we get $f(0)=0$. Replacing $y$ by $x$ and $2 x$ in (1) respectively, we arrive $f(2 x)=2 f(x)$ and $f(3 x)=3 f(x)$, respectively for all $x \in E$. In general, for any positive integer $a$, we obtain

$f(a x)=a f(x)$

for all $x \in E$. Substituting $x$ by $\frac{x}{a}$ in (7), we have

$f\left(\frac{x}{a}\right)=\frac{1}{a} f(x)$

for all $x \in E$. Replacing $(x, y)$ by $\left(x_{0}, x_{1}\right)$ in (1), we get

$f\left(x_{0}+x_{1}\right)=f\left(x_{0}\right)+f\left(x_{1}\right)$

for all $x_{0}, x_{1} \in E$. Again replacing $(x, y)$ by $\left(x_{0},-x_{1}\right)$ in $(1)$, we get

$f\left(x_{0}-x_{1}\right)=f\left(x_{0}\right)+f\left(-x_{1}\right)$

for all $x_{0}, x_{1} \in E$. Adding (9) and (10), we arrive

$f\left(x_{0}+x_{1}\right)+f\left(x_{0}-x_{1}\right)=2 f\left(x_{0}\right)+f\left(x_{1}\right)+f\left(-x_{1}\right)$

for all $x_{0}, x_{1} \in E$. Substituting $\left(x_{0}, x_{1}\right)$ by $\left(x_{2}, x_{3}\right)$ in (11), we get

$f\left(x_{2}+x_{3}\right)+f\left(x_{2}-x_{3}\right)=2 f\left(x_{2}\right)+f\left(x_{3}\right)+f\left(-x_{3}\right)$

for all $x_{2}, x_{3} \in E$. Again substituting $\left(x_{0}, x_{1}\right)$ by $\left(x_{4}, x_{5}\right)$ in (11), we obtain

$f\left(x_{4}+x_{5}\right)+f\left(x_{4}-x_{5}\right)=2 f\left(x_{4}\right)+f\left(x_{5}\right)+f\left(-x_{5}\right)$

for all $x_{4}, x_{5} \in E$. Continuing this process, finally replacing $\left(x_{0}, x_{1}\right)$ by $\left(x_{2 n}, x_{2 n+1}\right)$ in (11), we arrive

$f\left(x_{2 n}+x_{2 n+1}\right)+f\left(x_{2 n}-x_{2 n+1}\right)=2 f\left(x_{2 n}\right)+f\left(x_{2 n+1}\right)+f\left(-x_{2 n+1}\right)$

for all $x_{2 n}, x_{2 n+1} \in E$. Adding all the $n$ equations from (11) to (14), we desired our result.

Conversely, let $f: E \rightarrow F$ satisfies the functional equation (6). Letting $x_{0}=\ldots=x_{2 n+1}=0$ in (6), we get $f(0)=0$. Replacing $\left(x_{0}, x_{1}, x_{2}, \ldots, x_{2 n}, x_{2 n+1}\right)$ by $(x, y, 0, \ldots, 0,0)$, we get

$f(x+y)+f(x-y)=2 f(x)+f(y)+f(-y)$

for all $x, y \in E$. Using oddness of $f$ in (15), we have

$f(x+y)+f(x-y)=2 f(x)$

for all $x, y \in E$. By Theorem 2.1 of [4], we desired our result. Hence the proof is complete.

Lemma 2.2 If an even mapping $f: E \rightarrow F$ satisfies the functional equation (2) if and only if $f: E \rightarrow F$ satisfies the functional equation (6).

Proof. Assume $f: E \rightarrow F$ satisfies the functional equation (2). Letting $x=y=0$ in (2), we get $f(0)=0$. Setting $y$ by $x$ and $2 x$ in (2) respectively, we arrive $f(2 x)=4 f(x)$ and $f(3 x)=9 f(x)$ for all $x \in E$. In general, for any positive integer $b$, we arrive

$f(b x)=b^{2} f(x)$ 
for all $x \in E$. Substituting $x$ by $\frac{x}{b}$ in (17), we have

$f\left(\frac{x}{b}\right)=\frac{1}{b^{2}} f(x)$

for all $x \in E$. Replacing $(x, y)$ by $\left(x_{0}, x_{1}\right)$ in $(2)$, we get

$f\left(x_{0}+x_{1}\right)+f\left(x_{0}-x_{1}\right)=2 f\left(x_{0}\right)+2 f\left(x_{1}\right)$

for all $x_{0}, x_{1} \in E$. With the help of evenness of $f$, (19) becomes

$f\left(x_{0}+x_{1}\right)+f\left(x_{0}-x_{1}\right)=2 f\left(x_{0}\right)+f\left(x_{1}\right)+f\left(-x_{1}\right)$

for all $x_{0}, x_{1} \in E$. Substituting $\left(x_{0}, x_{1}\right)$ by $\left(x_{2}, x_{3}\right)$ in (20), we get

$f\left(x_{2}+x_{3}\right)+f\left(x_{2}-x_{3}\right)=2 f\left(x_{2}\right)+f\left(x_{3}\right)+f\left(-x_{3}\right)$

for all $x_{2}, x_{3} \in E$. Again substituting $\left(x_{0}, x_{1}\right)$ by $\left(x_{4}, x_{5}\right)$ in $(20)$, we obtain

$f\left(x_{4}+x_{5}\right)+f\left(x_{4}-x_{5}\right)=2 f\left(x_{4}\right)+f\left(x_{5}\right)+f\left(-x_{5}\right)$

for all $x_{4}, x_{5} \in E$. Continuing this process, finally replacing $\left(x_{0}, x_{1}\right)$ by $\left(x_{2 n}, x_{2 n+1}\right)$ in $(20)$, we arrive

$f\left(x_{2 n}+x_{2 n+1}\right)+f\left(x_{2 n}-x_{2 n+1}\right)=2 f\left(x_{2 n}\right)+f\left(x_{2 n+1}\right)+f\left(-x_{2 n+1}\right)$

for all $x_{2 n}, x_{2 n+1} \in E$. Adding all the $n$ equations from (20) to (23), we desired our result.

Conversely, let $f: E \rightarrow F$ satisfies the functional equation (6). Letting $x_{0}=x_{1}=\ldots x_{2 n}=x_{2 n+1}=0$ in $(6)$, we get $f(0)=0$. Replacing $\left(x_{0}, x_{1}, x_{2}, \ldots, x_{2 n}, x_{2 n+1}\right)$ by $(x, y, 0, \ldots, 0,0)$, we get

$f(x+y)+f(x-y)=2 f(x)+f(y)+f(-y)$

for all $x, y \in E$. Using evenness of $f$ in (24), we desired our result. Hence the proof is complete.

\section{Stability results: A direct method}

In section, let us take $E$ be a normed space and $F$ be a Banach space. Define a mapping $D f: E \rightarrow F$ by

$D f\left(x_{0}, x_{1}, \cdots, x_{2 n}, x_{2 n+1}\right)=\sum_{i=0}^{n}\left[f\left(x_{2 i}+x_{2 i+1}\right)+f\left(x_{2 i}-x_{2 i+1}\right)\right]-\sum_{i=0}^{n}\left[2 f\left(x_{2 i}\right)+f\left(x_{2 i+1}\right)+f\left(-x_{2 i+1}\right)\right]$

for all $x_{0}, x_{1}, \cdots, x_{2 n}, x_{2 n+1} \in E$.

In this section, we investigate the generalized Ulam-Hyers stability of the functional equation (6).

Theorem 3.1 Let $j= \pm 1$. Let $f_{a}: E \rightarrow F$ be an odd mapping for which there exist a function $\zeta: E^{2 n+1} \rightarrow[0, \infty)$ with the condition

$\lim _{m \rightarrow \infty} \frac{1}{2^{m j}} \zeta\left(2^{m j} x_{0}, 2^{m j} x_{1}, \cdots, 2^{m j} x_{2 n}, 2^{m j} x_{2 n+1}\right)=0$

such that the functional inequality

$\left\|D f_{a}\left(x_{0}, \cdots, x_{2 n+1}\right)\right\| \leq \zeta\left(x_{0}, x_{1}, \cdots, x_{2 n}, x_{2 n+1}\right)$

for all $x_{0}, \cdots, x_{2 n+1} \in E$. Then there exists a unique additive mapping $A: E \rightarrow F$ satisfying the functional equation (6) and

$\left\|f_{a}(x)-A(x)\right\| \leq \frac{1}{2} \sum_{k=\frac{1-j}{2}}^{\infty} \frac{1}{2^{k j}} \xi\left(2^{k j} x\right)$

for all $x \in E$, where $\xi\left(2^{k j} x\right)$ and $A(x)$ are defined by

$\xi\left(2^{k j} x\right)=\frac{1}{2 n} \zeta\left(2^{k j} x, 2^{k j} x, \cdots, 2^{k j} x, 2^{k j} x\right)$

and

$A(x)=\lim _{k \rightarrow \infty} \frac{1}{2^{k j}} f_{a}\left(2^{k j} x\right)$

for all $x \in E$. 
Proof. Assume $j=1$. Replacing $\left(x_{0}, x_{1}, \cdots, x_{2 n}, x_{2 n+1}\right)$ by $(x, x, \cdots, x, x)$ in (26) and using oddness of $f_{a}$, we get

$\left\|2 n f_{a}(2 x)-4 n f_{a}(x)\right\| \leq \zeta(x, x, \cdots, x, x)$

for all $x \in E$. It follows from (30) that

$\left\|\frac{f_{a}(2 x)}{2}-f_{a}(x)\right\| \leq \frac{\xi(x)}{2} \quad$ where $\xi(x)=\frac{1}{2 n} \zeta(x, x, \cdots, x, x)$

for all $x \in E$. Now replacing $x$ by $2 x$ and dividing by 2 in (31), we get

$\left\|\frac{f_{a}\left(2^{2} x\right)}{2^{2}}-\frac{f_{a}(2 x)}{2}\right\| \leq \frac{\xi(2 x)}{2^{2}}$

for all $x \in E$. From (31) and (32), we obtain

$\left\|\frac{f_{a}\left(2^{2} x\right)}{2^{2}}-f_{a}(x)\right\| \leq\left\|\frac{f_{a}(2 x)}{2}-f_{a}(x)\right\|+\left\|\frac{f_{a}\left(2^{2} x\right)}{2^{2}}-\frac{f_{a}(2 x)}{2}\right\| \leq \frac{1}{2}\left[\xi(x)+\frac{\xi(2 x)}{2}\right]$

for all $x \in E$. Continuing this process after $m$ times, one can arrive

$\left\|\frac{f_{a}\left(2^{m} x\right)}{2^{m}}-f_{a}(x)\right\| \leq \frac{1}{2} \sum_{k=0}^{m-1} \frac{\xi\left(2^{k} x\right)}{2^{k}} \leq \frac{1}{2} \sum_{k=0}^{\infty} \frac{\xi\left(2^{k} x\right)}{2^{k}}$

for all $x \in E$. In order to prove the convergence of the sequence $\left\{\frac{f_{a}\left(2^{m} x\right)}{2^{m}}\right\}$, replace $x$ by $2^{l} x$ and dividing by $2^{l}$ in (34), for any $m, l>0$, we deduce

$\left\|\frac{f_{a}\left(2^{m+l} x\right)}{2^{(m+l)}}-\frac{f_{a}\left(2^{l} x\right)}{2^{l}}\right\|=\frac{1}{2^{l}}\left\|\frac{f_{a}\left(2^{m} \cdot 2^{l} x\right)}{2^{m}}-f_{a}\left(2^{l} x\right)\right\| \leq \frac{1}{2} \sum_{k=0}^{\infty} \frac{\xi\left(2^{k+l} x\right)}{2^{k+l}} \rightarrow 0 \quad$ as $m \rightarrow \infty$

for all $x \in E$. Hence the sequence $\left\{\frac{f_{a}\left(2^{m} x\right)}{2^{m}}\right\}$ is Cauchy sequence. Since $F$ is complete, there exists a mapping $A: E \rightarrow F$ such that

$$
A(x)=\lim _{m \rightarrow \infty} \frac{f_{a}\left(2^{m} x\right)}{2^{m}} \forall x \in E .
$$

Letting $m \rightarrow \infty$ in (34) we see that (27) holds for all $x \in E$. To prove that $A$ satisfies (6), replacing $\left(x_{0}, x_{1}, \cdots, x_{2 n}, x_{2 n+1}\right)$ by $\left(2^{m} x_{0}, 2^{m} x_{1}, \cdots, 2^{m} x_{2 n}, 2^{m} x_{2 n+1}\right)$ and dividing by $2^{m}$ in (26), we obtain

$\frac{1}{2^{m}}\left\|D f_{a}\left(2^{m} x_{0}, 2^{m} x_{1}, \cdots, 2^{m} x_{2 n}, 2^{m} x_{2 n+1}\right)\right\| \leq \frac{1}{2^{m}} \zeta\left(2^{m} x_{0}, 2^{m} x_{1}, \cdots, 2^{m} x_{2 n}, 2^{m} x_{2 n+1}\right)$

for all $x_{0}, x_{1}, \cdots, x_{2 n}, x_{2 n+1} \in E$. Letting $m \rightarrow \infty$ in the above inequality and using the definition of $A(x)$, we see that

$D A\left(x_{0}, x_{1}, \cdots, x_{2 n}, x_{2 n+1}\right)=0$.

Hence $A$ satisfies (6) for all $x_{0}, x_{1}, \cdots, x_{2 n}, x_{2 n+1} \in E$. To prove that $A$ is unique, let $B(x)$ be another additive mapping satisfying (6) and (27), then

$$
\|A(x)-B(x)\| \leq \frac{1}{2^{m}}\left\{\left\|A\left(2^{m} x\right)-f_{a}\left(2^{m} x\right)\right\|+\left\|f_{a}\left(2^{m} x\right)-B\left(2^{m} x\right)\right\|\right\} \leq \sum_{k=0}^{\infty} \frac{\xi\left(2^{k+m} x\right)}{2^{(k+m)}} \rightarrow 0 \quad \text { as } m \rightarrow \infty
$$

for all $x \in E$. Hence $A$ is unique.

For $j=-1$, we can prove a similar stability result. This completes the proof of the theorem.

The following Corollary is an immediate consequence of Theorem 3.1 concerning the stability of (6). 
Corollary 3.2 Let $\rho$ and $s$ be nonnegative real numbers. Let an odd function $f_{a}: E \rightarrow F$ satisfies the inequality $\left\|D f_{a}\left(x_{0}, \cdots, x_{2 n+1}\right)\right\| \leq\left\{\begin{array}{lll}\rho,\left\{\sum_{i=0}^{2 n+1}\left\|x_{i}\right\|^{s}\right\}, & s<1 \quad \text { or } & s>1 ; \\ \rho\left\{\prod_{i=0}^{2 n+1}\left\|x_{i}\right\|^{s}\right\}, & s<\frac{1}{(2 n+1)} & \text { or } \quad s>\frac{1}{(2 n+1)} \\ \rho\left\{\prod_{i=0}^{2 n+1}\left\|x_{i}\right\|^{s}+\left\{\sum_{i=0}^{2 n+1}\left\|x_{i}\right\|^{s}\right\}\right\}, & s<\frac{1}{(2 n+1)} & \text { or } \quad s>\frac{1}{(2 n+1)} ;\end{array}\right.$

for all $x_{0}, x_{1}, \cdots, x_{2 n}, x_{2 n+1} \in E$. Then there exists a unique additive function $A: E \rightarrow F$ such that

$\left\|f_{a}(x)-A(x)\right\| \leq\left\{\begin{array}{l}\frac{\rho}{2 n}, \\ \frac{(2 n+1) \rho\|x\|^{s}}{2 n\left|2-2^{s}\right|} \\ \frac{\left.\rho\|x\|\right|^{(2 n+1) s}}{2 n\left|2-2^{(2 n+1) s}\right|} \\ \frac{(n+1) \rho|| x||^{(2 n+1) s}}{n\left|2-2^{(2 n+1) s}\right|}\end{array}\right.$

for all $x \in X$.

Theorem 3.3 Let $j= \pm 1$. Let $f_{q}: E \rightarrow F$ be an even mapping for which there exist a function $\zeta: E^{2 n+1} \rightarrow[0, \infty)$ with the condition

$\lim _{k \rightarrow \infty} \frac{1}{4^{m j}} \zeta\left(2^{m j} x_{0}, 2^{m j} x_{1}, \cdots, 2^{m j} x_{2 n}, 2^{m j} x_{2 n+1}\right)=0$

such that the functional inequality

$\left\|D f_{q}\left(x_{0}, x_{1}, \cdots, x_{2 n}, x_{2 n+1}\right)\right\| \leq \zeta\left(x_{0}, x_{1}, \cdots, x_{2 n}, x_{2 n+1}\right)$

for all $x_{0}, x_{1}, \cdots, x_{2 n}, x_{2 n+1} \in E$. Then there exists a unique quadratic mapping $Q: E \rightarrow F$ satisfying the functional equation (6) and

$\left\|f_{q}(x)-Q(x)\right\| \leq \frac{1}{4} \sum_{k=\frac{1-j}{2}}^{\infty} \frac{1}{4^{k j}} \xi\left(2^{k j} x\right)$

for all $x \in E$, where $\xi\left(2^{k j} x\right)$ and $Q(x)$ are defined by

$\xi\left(2^{k j} x\right)=\frac{1}{2 n} \zeta\left(2^{k j} x, 2^{k j} x, \cdots, 2^{k j} x, 2^{k j} x\right)$

and

$Q(x)=\lim _{k \rightarrow \infty} \frac{1}{4^{k j}} f_{q}\left(2^{k j} x\right)$

for all $x \in E$.

Proof. Assume $j=1$. Replacing $\left(x_{0}, x_{1}, \cdots, x_{2 n}, x_{2 n+1}\right)$ by $(x, x, \cdots, x, x)$ in (38) and using evenness of $f_{q}$, we get

$\left\|2 n f_{q}(2 x)-8 n f_{q}(x)\right\| \leq \zeta(x, x, \cdots, x, x)$

for all $x \in E$. It follows from (42) that

$\left\|\frac{f_{q}(2 x)}{4}-f_{q}(x)\right\| \leq \frac{\xi(x)}{4} \quad$ where $\xi(x)=\frac{1}{2 n} \zeta(x, x, \cdots, x, x)$

for all $x \in E$. The rest of the proof is similar tracing to that of Theorem 3.1.

The following Corollary is an immediate consequence of Theorem 3.3 concerning the stability of (6). 
Corollary 3.4 Let $\rho$ and $s$ be nonnegative real numbers. Let an even function $f_{q}: E \rightarrow F$ satisfies the inequality

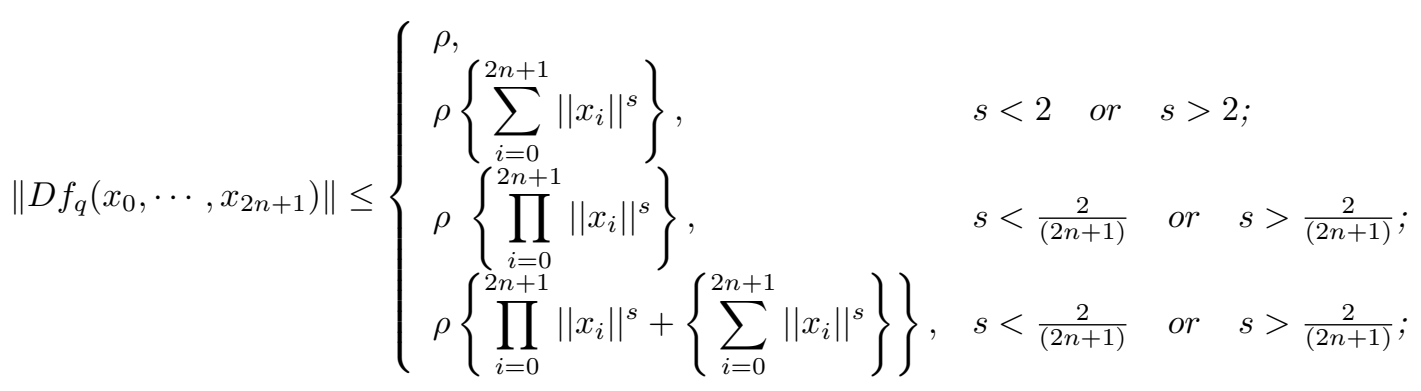

for all $x_{0}, x_{1}, \cdots, x_{2 n}, x_{2 n+1} \in E$. Then there exists a unique quadratic function $Q: X \rightarrow Y$ such that

$$
\left\|f_{q}(x)-Q(x)\right\| \leq\left\{\begin{array}{l}
\frac{\rho}{6 n}, \\
\frac{(2 n+1) \rho\|x\|^{s}}{2 n\left|4-2^{s}\right|} \\
\frac{\rho \| x||^{(2 n+1) s}}{2 n\left|4-2^{(2 n+1) s}\right|} \\
\frac{(n+1) \rho\|x\|^{(2 n+1) s}}{n\left|4-2^{(2 n+1) s}\right|}
\end{array}\right.
$$

for all $x \in E$.

Now we are ready to prove our mixed stability theorem.

Theorem 3.5 Let $j= \pm 1$. Let $f: E \rightarrow F$ be a mapping for which there exist a function $\zeta: E^{2 n+1} \rightarrow[0, \infty)$ with the condition (25) and (37) such that the functional inequality

$\left\|D f\left(x_{0}, \cdots, x_{2 n+1}\right)\right\| \leq \zeta\left(x_{0}, x_{1}, \cdots, x_{2 n}, x_{2 n+1}\right)$

for all $x_{0}, x_{1}, \cdots, x_{2 n}, x_{2 n+1} \in E$. Then there exists a unique additive mapping $A: E \rightarrow F$ and a unique quadratic mapping $Q: E \rightarrow F$ such that

$\|f(x)-A(x)-Q(x)\| \leq \frac{1}{2}\left[\frac{1}{2} \sum_{k=\frac{1-j}{2}}^{\infty}\left(\frac{\xi\left(2^{k j} x\right)}{2^{k j}}+\frac{\xi\left(-2^{k j} x\right)}{2^{k j}}\right)+\frac{1}{4} \sum_{k=\frac{1-j}{2}}^{\infty}\left(\frac{\xi\left(2^{k j} x\right)}{4^{k j}}+\frac{\xi\left(-2^{k j} x\right)}{4^{k j}}\right)\right]$

for all $x \in E$, where $\xi(x), A(x)$ and $Q(x)$ are defined in (28), (29) and (41) respectively for all $x \in E$.

Proof. Let $f_{o}(x)=\frac{f_{a}(x)-f_{a}(-x)}{2}$ for all $x \in E$. Then $f_{o}(0)=0$ and $f_{o}(-x)=-f_{o}(x)$ for all $x \in X$. Hence

$$
\begin{aligned}
\left\|D f_{o}\left(x_{0}, x_{1}, \cdots, x_{2 n}, x_{2 n+1}\right)\right\| & \leq \frac{1}{2}\left[\left\|D f_{a}\left(x_{0}, x_{1}, \cdots, x_{2 n}, x_{2 n+1}\right)\right\|+\left\|D f_{a}\left(x_{0}, x_{1}, \cdots, x_{2 n}, x_{2 n+1}\right)\right\|\right] \\
& \leq \frac{1}{2}\left[\zeta\left(x_{0}, x_{1}, \cdots, x_{2 n}, x_{2 n+1}\right)+\zeta\left(-x_{0},-x_{1}, \cdots,-x_{2 n},-x_{2 n+1}\right)\right]
\end{aligned}
$$

for all $x_{0}, x_{1}, \cdots, x_{2 n}, x_{2 n+1} \in E$. By Theorem 3.1, we have

$\left\|f_{o}(x)-A(x)\right\| \leq \frac{1}{4} \sum_{k=\frac{1-j}{2}}^{\infty}\left(\frac{\xi\left(2^{k j} x\right)}{2^{k j}}+\frac{\xi\left(-2^{k j} x\right)}{2^{k j}}\right)$

for all $x \in E$. Also, let $f_{e}(x)=\frac{f_{q}(x)+f_{q}(-x)}{2}$ for all $x \in E$. Then $f_{e}(0)=0$ and $f_{e}(-x)=f_{e}(x)$ for all $x \in x$. Hence

$$
\begin{aligned}
\left\|D f_{e}\left(x_{0}, x_{1}, \cdots, x_{2 n}, x_{2 n+1}\right)\right\| & \leq \frac{1}{2}\left[\left\|D f_{q}\left(x_{0}, x_{1}, \cdots, x_{2 n}, x_{2 n+1}\right)\right\|+\left\|D f_{q}\left(x_{0}, x_{1}, \cdots, x_{2 n}, x_{2 n+1}\right)\right\|\right] \\
& \leq \frac{1}{2}\left[\zeta\left(x_{0}, x_{1}, \cdots, x_{2 n}, x_{2 n+1}\right)+\zeta\left(-x_{0},-x_{1}, \cdots,-x_{2 n},-x_{2 n+1}\right)\right]
\end{aligned}
$$


for all $x_{0}, x_{1}, \cdots, x_{2 n}, x_{2 n+1} \in E$. By Theorem 3.3, we have

$$
\left\|f_{e}(x)-Q(x)\right\| \leq \frac{1}{8} \sum_{k=\frac{1-j}{2}}^{\infty}\left(\frac{\xi\left(2^{k j} x\right)}{4^{k j}}+\frac{\xi\left(-2^{k j} x\right)}{4^{k j}}\right)
$$

for all $x \in E$. Define

$f(x)=f_{e}(x)+f_{o}(x)$

for all $x \in E$. From (49),(51) and (52), we arrive

$$
\begin{aligned}
\|f(x)-A(x)-Q(x)\| & =\left\|f_{e}(x)+f_{o}(x)-A(x)-Q(x)\right\| \\
& \leq\left\|f_{o}(x)-A(x)\right\|+\left\|f_{e}(x)-Q(x)\right\| \\
& \leq \frac{1}{4} \sum_{k=\frac{1-j}{2}}^{\infty}\left(\frac{\xi\left(2^{k j} x\right)}{2^{k j}}+\frac{\xi\left(-2^{k j} x\right)}{2^{k j}}\right)+\frac{1}{8} \sum_{k=\frac{1-j}{2}}^{\infty}\left(\frac{\xi\left(2^{k j} x\right)}{4^{k j}}+\frac{\xi\left(-2^{k j} x\right)}{4^{k j}}\right)
\end{aligned}
$$

for all $x \in E$. Hence the theorem is proved.

Using Corollaries 3.2 and 3.4 we have the following Corollary concerning the stability of (6).

Corollary 3.6 Let $\rho$ and $s$ be nonnegative real numbers. Let a function $f: E \rightarrow F$ satisfies the inequality

$$
\left\|D f\left(x_{0}, \cdots, x_{2 n+1}\right)\right\| \leq \begin{cases}\rho\left\{\sum_{i=0}^{\rho,}\left\|x_{i}\right\|^{s}\right\}, & s \neq 1,2 ; \\ \rho\left\{\prod_{i=0}^{2 n+1}\left\|x_{i}\right\|^{s}\right\}, & s \neq \frac{1}{(2 n+1)}, \frac{2}{(2 n+1)} ; \\ \rho\left\{\prod_{i=0}^{2 n+1}\left\|x_{i}\right\|^{s}+\left\{\sum_{i=0}^{2 n+1}\left\|x_{i}\right\|^{s}\right\}\right\}, & s \neq \frac{1}{(2 n+1)}, \frac{2}{(2 n+1)} ;\end{cases}
$$

for all $x_{0}, x_{1}, \cdots, x_{2 n}, x_{2 n+1} \in E$. Then there exists a unique additive function $A: E \rightarrow F$ and a unique quadratic function $Q: E \rightarrow F$ such that

$$
\|f(x)-A(x)-Q(x)\| \leq\left\{\begin{array}{l}
\rho\left(\frac{1}{2 n}+\frac{1}{6 n}\right), \\
(2 n+1) \rho\|x\|^{s}\left(\frac{1}{n\left|2-2^{s}\right|}+\frac{1}{n\left|4-2^{s}\right|}\right), \\
\rho\|x\|^{(2 n+1) s}\left(\frac{1}{n\left|2-2^{(2 n+1) s}\right|}+\frac{1}{n\left|4-2^{(2 n+1) s}\right|}\right), \\
(n+1) \rho\|x\|^{(2 n+1) s}\left(\frac{1}{n\left|2-2^{(2 n+1) s}\right|}+\frac{1}{n\left|4-2^{(2 n+1) s}\right|}\right)
\end{array}\right.
$$

for all $x \in E$.

\section{Stability results: fixed point method}

In this section, we apply a fixed point method for achieving stability of the $n$ dimensional AQ - mixed type functional equation (6).

Now, we present the following theorem due to B. Margolis and J.B. Diaz [20] for fixed point Theory.

Theorem 4.1 [20] Suppose that for a complete generalized metric space $(\Omega, \delta)$ and a strictly contractive mapping $T: \Omega \rightarrow \Omega$ with Lipschitz constant $L$. Then, for each given $x \in \Omega$, either

$$
d\left(T^{n} x, T^{n+1} x\right)=\infty \quad \forall \quad n \geq 0,
$$

or there exists a natural number $n_{0}$ such that

(FP1) $d\left(T^{n} x, T^{n+1} x\right)<\infty$ for all $n \geq n_{0}$;

(FP2) The sequence $\left(T^{n} x\right)$ is convergent to a fixed to a fixed point $y^{*}$ of $T$

(FP3) $y^{*}$ is the unique fixed point of $T$ in the set $\Delta=\left\{y \in \Omega: d\left(T^{n_{0}} x, y\right)<\infty\right\}$;

(FP4) $d\left(y^{*}, y\right) \leq \frac{1}{1-L} d(y, T y)$ for all $y \in \Delta$. 
Using the above theorem, we now obtain the generalized Ulam - Hyers stability of (6).

Theorem 4.2 Let $f_{a}: E \rightarrow F$ be a odd mapping for which there exist a function $\zeta: E^{2 n+1} \rightarrow[0, \infty)$ with the condition

$\lim _{k \rightarrow \infty} \frac{1}{\kappa_{i}^{k}} \zeta\left(\kappa_{i}^{k} x_{0}, \kappa_{i}^{k} x_{1}, \cdots, \kappa_{i}^{k} x_{2 n}, \kappa_{i}^{k} x_{2 n+1}\right)=0$

where

$\kappa_{i}=\left\{\begin{array}{lll}2 & \text { if } & i=0 \\ \frac{1}{2} & \text { if } & i=1\end{array}\right.$

such that the functional inequality

$\left\|D f_{a}\left(x_{0}, x_{1}, \cdots, x_{2 n}, x_{2 n+1}\right)\right\| \leq \zeta\left(x_{0}, x_{1}, \cdots, x_{2 n}, x_{2 n+1}\right)$

for all $x_{0}, x_{1}, \cdots, x_{2 n}, x_{2 n+1} \in E$. If there exists $L=L(i)<1$ such that the function

$$
x \rightarrow \psi(x)=\xi\left(\frac{x}{2}\right),
$$

has the property

$\psi(x)=L \kappa_{i} \psi\left(\kappa_{i} x\right)$.

for all $x \in E$. Then there exists a unique additive mapping $A: E \rightarrow F$ satisfying the functional equation (6) and

$\left\|f_{a}(x)-A(x)\right\| \leq \frac{L^{1-i}}{1-L} \psi(x)$

for all $x \in E$.

Proof. Consider the set

$$
\Gamma=\{p / p: E \rightarrow F, p(0)=0\}
$$

and introduce the generalized metric on $\Gamma$,

$$
d(p, q)=\inf \{K \in(0, \infty):\|p(x)-q(x)\| \leq K \psi(x), x \in E\} .
$$

It is easy to see that $(\Gamma, d)$ is complete.

Define $\Upsilon: \Gamma \rightarrow \Gamma$ by

$$
\Upsilon p(x)=\frac{1}{\kappa_{i}} p\left(\kappa_{i} x\right)
$$

for all $x \in E$. Now $p, q \in \Gamma$,

$$
\begin{aligned}
d(p, q) \leq K & \Rightarrow\|p(x)-q(x)\| \leq K \psi(x), x \in E . \\
& \Rightarrow\left\|\frac{1}{\kappa_{i}} p\left(\kappa_{i} x\right)-\frac{1}{\kappa_{i}} q\left(\kappa_{i} x\right)\right\| \leq \frac{1}{\kappa_{i}} K \psi\left(\kappa_{i} x\right), x \in E, \\
& \Rightarrow\left\|\frac{1}{\kappa_{i}} p\left(\kappa_{i} x\right)-\frac{1}{\kappa_{i}} q\left(\kappa_{i} x\right)\right\| \leq L K \psi(x), x \in E, \\
& \Rightarrow\|\Upsilon p(x)-\Upsilon q(x)\| \leq L K \psi(x), x \in E, \\
& \Rightarrow d(p, q) \leq L K .
\end{aligned}
$$

This implies $d(\Upsilon p, \Upsilon q) \leq L d(p, q)$, for all $p, q \in \Gamma$. i.e., $T$ is a strictly contractive mapping on $\Gamma$ with Lipschitz constant $L$.

From (31), we arrive

$\left\|\frac{f_{a}(2 x)}{2}-f_{a}(x)\right\| \leq \frac{\xi(x)}{2}$ 
where

$$
\xi(x)=\frac{1}{2 n} \zeta(x, x, \cdots, x, x)
$$

for all $x \in E$. Using (58) for the case $i=0$ it reduces to

$\left\|\frac{f_{a}(2 x)}{2}-f_{a}(x)\right\| \leq L \psi(x)$

for all $x \in E$,

$$
\text { i.e., } \quad d\left(\Upsilon f_{a}, f_{a}\right) \leq L \Rightarrow d\left(\Upsilon f_{a}, f_{a}\right) \leq L=L^{1}<\infty .
$$

Again replacing $x=\frac{x}{2}$ in (60), we get

$\left\|f_{a}(x)-2 f_{a}\left(\frac{x}{2}\right)\right\| \leq \xi\left(\frac{x}{2}\right)$

Using (58) for the case $i=1$ it reduces to

$\left\|f_{a}(x)-2 f_{a}\left(\frac{x}{2}\right)\right\| \leq \psi(x)$

for all $x \in E$,

$$
\text { i.e., } \quad d\left(f_{a}, \Upsilon f_{a}\right) \leq 1 \Rightarrow d\left(f_{a}, \Upsilon f_{a}\right) \leq 1=L^{0}<\infty .
$$

From the above two cases, we arrive

$$
d\left(f_{a}, \Upsilon f_{a}\right) \leq L^{1-i}
$$

Therefore (FP1) holds.

By (FP2), it follows that there exists a fixed point $A$ of $\Upsilon$ in $\Gamma$ such that

$A(x)=\lim _{k \rightarrow \infty} \frac{f_{a}\left(\kappa_{i}^{k} x\right)}{\kappa_{i}^{k}}, \quad \forall x \in E$

To order to prove $A: E \rightarrow F$ is additive. Replacing $\left(x_{0}, \cdots, x_{2 n+1}\right)$ by $\left(\kappa_{i}^{k} x_{0}, \cdots, \kappa_{i}^{k} x_{2 n+1}\right)$ in $(57)$ and dividing by $\kappa_{i}^{k}$, it follows from (55) that

$\frac{1}{\kappa_{i}^{k}}\left\|D f_{a}\left(\kappa_{i}^{k} x_{0}, \kappa_{i}^{k} x_{1}, \cdots, \kappa_{i}^{k} x_{2 n}, \kappa_{i}^{k} x_{2 n+1}\right)\right\| \leq \frac{1}{\kappa_{i}^{k}} \zeta\left(\kappa_{i}^{k} x_{0}, \kappa_{i}^{k} x_{1}, \cdots, \kappa_{i}^{k} x_{2 n}, \kappa_{i}^{k} x_{2 n+1}\right)$

for all $x_{0}, x_{1}, \cdots, x_{2 n}, x_{2 n+1} \in E$. Letting $k \rightarrow \infty$ in the above inequality and using the definition of $A(x)$, we see that

$$
D A\left(x_{0}, x_{1}, \cdots, x_{2 n}, x_{2 n+1}\right)=0
$$

i.e., $A$ satisfies the functional equation (6) for all $x_{0}, x_{1}, \cdots, x_{2 n}, x_{2 n+1} \in E$.

By (FP3), $A$ is the unique fixed point of $\Upsilon$ in the set

$$
\Delta=\left\{A \in \Gamma: d\left(f_{a}, A\right)<\infty\right\}
$$

such that

$$
\left\|f_{a}(x)-A(x)\right\| \leq K \psi(x)
$$

for all $x \in E$ and $K>0$. Finally by (FP4), we obtain

$$
d\left(f_{a}, A\right) \leq \frac{1}{1-L} d\left(f_{a}, \Upsilon f_{a}\right)
$$

this implies

$$
d\left(f_{a}, A\right) \leq \frac{L^{1-i}}{1-L}
$$

which yields

$$
\left\|f_{a}(x)-A(x)\right\| \leq \frac{L^{1-i}}{1-L} \psi(x)
$$

this completes the proof of the theorem.

The following Corollary is an immediate consequence of Theorem 4.2 concerning the stability of (6). 
Corollary 4.3 Let $f_{a}: X \rightarrow Y$ be a mapping and there exists real numbers $\rho$ and $s$ such that

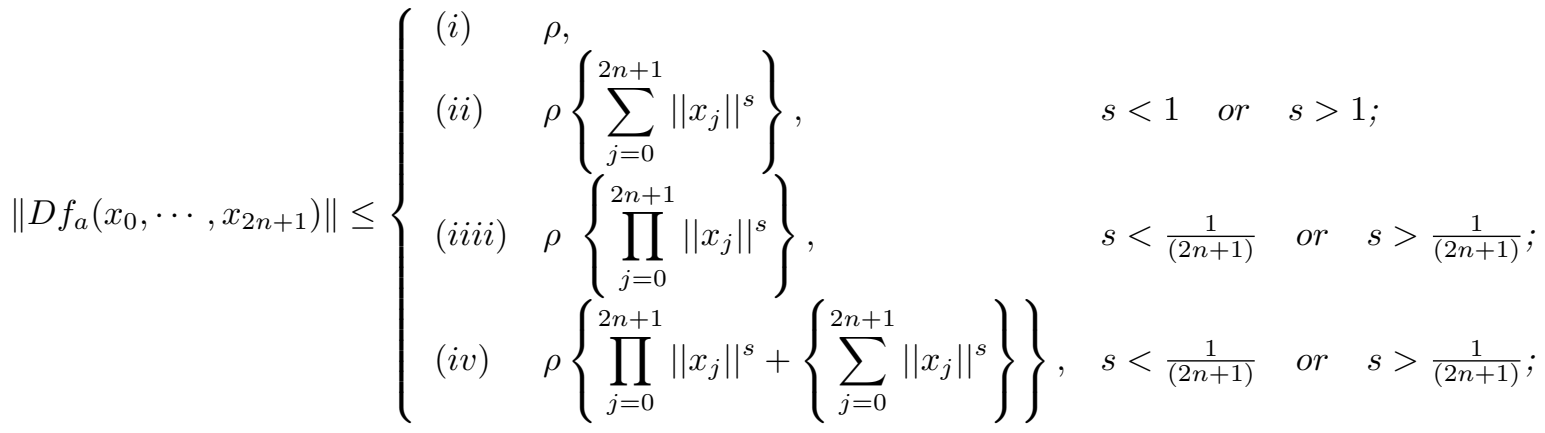

for all $x_{0}, x_{1}, \cdots, x_{2 n}, x_{2 n+1} \in E$. Then there exists a unique additive function $A: E \rightarrow F$ such that

$\left\|f_{a}(x)-A(x)\right\| \leq \begin{cases}(i) & \frac{|\rho|}{n}, \\ (i i) & \frac{(2 n+1) \rho\|x\|^{s}}{n\left|2-2^{s}\right|}, \\ \text { (iii) } & \frac{\rho \|\left. x\right|^{(2 n+1) s}}{n \mid 2-2^{(2 n+1) s \mid}}, \\ \text { (iv }) & \frac{\left.(2 n+2) \rho\|x\|\right|^{(2 n+1) s}}{n\left|2-2^{(2 n+1) s}\right|},\end{cases}$

for all $x \in E$.

Proof. Setting

$\zeta\left(x_{0}, x_{1}, \cdots, x_{2 n}, x_{2 n+1}\right)=\left\{\begin{array}{l}\rho,\left\{\sum_{j=0}^{2 n+1}\left\|x_{j}\right\|^{s}\right\}, \\ \rho\left\{\prod_{j=0}^{2 n+1}\left\|x_{j}\right\|^{s}\right\}, \\ \rho\left\{\prod_{j=0}^{2 n+1}\left\|x_{j}\right\|^{s}+\left\{\sum_{j=0}^{2 n+1}\left\|x_{j}\right\|^{s}\right\}\right\},\end{array}\right.$

for all $x_{0}, x_{1}, \cdots, x_{2 n}, x_{2 n+1} \in E$. Now,

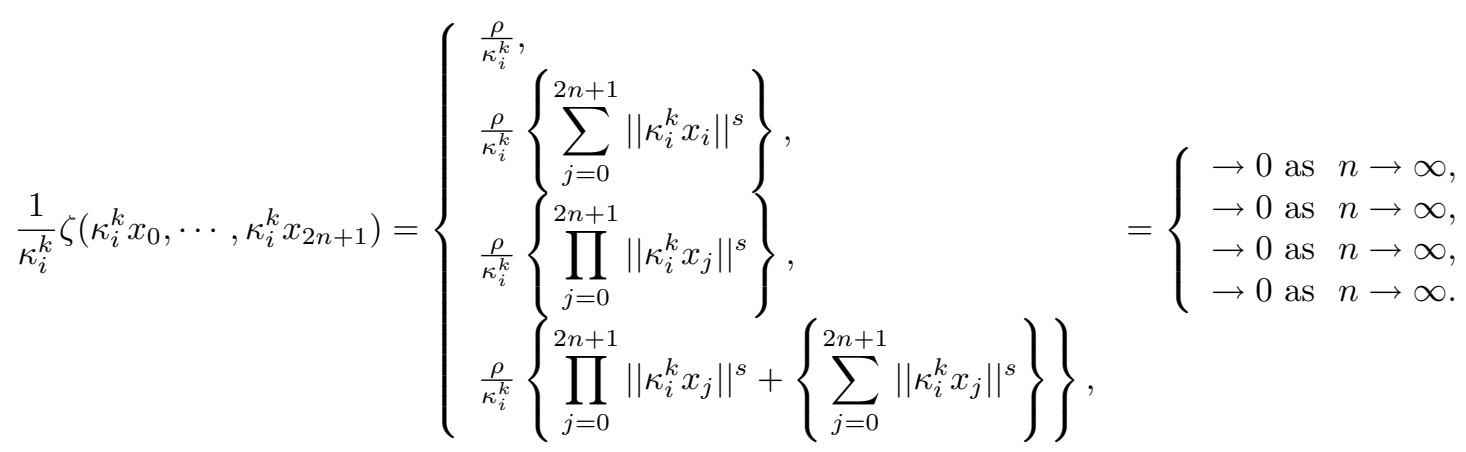

Thus, (55) is holds.

But we have $\psi(x)=\xi\left(\frac{x}{2}\right)$ has the property $\psi(x)=L \cdot \kappa_{i} \psi\left(\kappa_{i} x\right)$ for all $x \in E$. Hence

$\psi(x)=\xi\left(\frac{x}{2}\right)=\frac{1}{2 n} \zeta\left(\frac{x}{2}, \frac{x}{2}, \cdots, \frac{x}{2}, \frac{x}{2}\right)=\left\{\begin{array}{l}\frac{\rho}{n} \\ \frac{(2 n+1) \rho}{2^{s} n}\|x\|^{s}, \\ \frac{\rho}{2^{(2 n+1) s} n}\|x\|^{(2 n+1) s}, \\ \frac{(2 n+2) \rho}{2^{(2 n+1) s} n}\|x\|^{(2 n+1) s} .\end{array}\right.$ 
Now,

$$
\begin{aligned}
\frac{1}{\kappa_{i}} \psi\left(\kappa_{i} x\right)=\left\{\begin{array}{l}
\frac{\rho}{\kappa_{i} n} \\
\frac{(2 n+1) \rho}{\kappa_{i} 2^{s} n}\left\|\kappa_{i} x\right\|^{s}, \\
\frac{\rho}{\kappa_{i} 2^{(2 n+1) s} n}\left\|\kappa_{i} x\right\|^{(2 n+1) s}, \\
\frac{(2 n+2) \rho}{\kappa_{i} 2^{(2 n+1) s} n}\left\|\kappa_{i} x\right\|^{(2 n+1) s} .
\end{array}=\left\{\begin{array}{l}
\kappa_{i}^{-1} \frac{\rho}{n}, \\
\kappa_{i}^{s-1} \frac{(2 n+1) \rho}{2^{s} n}\|x\|^{s} \\
\kappa_{i}^{(2 n+1) s-1} \frac{\rho}{2^{(2 n+1) s} n}\|x\|^{(2 n+1) s} \\
\kappa_{i}^{(2 n+1) s-1} \frac{(2 n+2) \rho}{2^{(2 n+1) s} n}\|x\|^{(2 n+1) s} .
\end{array}\right.\right. \\
=\left\{\begin{array}{l}
\kappa_{i}^{-1} \psi(x), \\
\kappa_{i}^{s-1} \psi(x), \\
\kappa_{i}^{(2 n+1) s-1} \psi(x), \\
\kappa_{i}^{(2 n+1) s-1} \psi(x) .
\end{array}\right.
\end{aligned}
$$

Hence the inequality (58) holds either, $L=2^{-1}$ for $s=0$ if $i=0$ and $L=\frac{1}{2^{-1}}$ for $s=0$ if $i=1$. Now from (59), we prove the following cases for condition $(i)$.

Case: $1 L=2^{-1}$ for $s=0$ if $i=0$

$\left\|f_{a}(x)-A(x)\right\| \leq \frac{\left(2^{-1}\right)^{1-0}}{1-2^{-1}} \psi(x)=\frac{\rho}{n}$.

Case:2 $L=\frac{1}{2^{-1}}$ for $s=0$ if $i=1$

$\left\|f_{a}(x)-A(x)\right\| \leq \frac{\left(\frac{1}{2^{-1}}\right)^{1-1}}{1-\frac{1}{2^{-1}}} \psi(x)=\frac{-\rho}{n}$.

Also the inequality (58) holds either, $L=2^{s-1}$ for $s<1$ if $i=0$ and $L=\frac{1}{2^{s-1}}$ for $s>1$ if $i=1$. Now from (59), we prove the following cases for condition (ii).

Case: $1 L=2^{s-1}$ for $s<1$ if $i=0$

$\left\|f_{a}(x)-A(x)\right\| \leq \frac{\left(2^{(s-1)}\right)^{1-0}}{1-2^{(s-1)}} \psi(x)=\frac{1}{2-2^{s}} \frac{(2 n+1) \rho}{n}\|x\|^{s}=\frac{(2 n+1) \rho\|x\|^{s}}{n\left(2-2^{s}\right)}$.

Case:2 $L=\frac{1}{2^{s-1}}$ for $s>1$ if $i=1$

$\left\|f_{a}(x)-A(x)\right\| \leq \frac{\left(\frac{1}{2^{(s-1)}}\right)^{1-1}}{1-\frac{1}{2^{(s-1)}}} \psi(x)=\frac{1}{2^{s}-2} \frac{(2 n+1) \rho}{n}\|x\|^{s}=\frac{(2 n+1) \rho\|x\|^{s}}{n\left(2^{s}-2\right)}$.

Again, the inequality (58) holds either, $L=2^{(2 n+1) s-1}$ for $s<\frac{1}{(2 n+1)}$ if $i=0$ and $L=\frac{1}{2^{(2 n+1) s-1}}$ for $s>\frac{1}{(2 n+1)}$ if $i=1$. Now from (59), we prove the following cases for condition (iii).

Case: $1 L=2^{(2 n+1) s-1}$ for $s<\frac{1}{(2 n+1)}$ if $i=0$

$\left\|f_{a}(x)-A(x)\right\| \leq \frac{\left(2^{((2 n+1) s-1)}\right)^{1-0}}{1-2^{((2 n+1) s-1)}} \psi(x)=\frac{1}{2-2^{(2 n+1) s}} \frac{\rho}{n}\|x\|^{(2 n+1) s}=\frac{\rho\|x\|^{(2 n+1) s}}{n\left(2-2^{(2 n+1) s}\right)}$.

Case:2 $L=\frac{1}{2^{(2 n+1) s-1}}$ for $s>\frac{1}{(2 n+1)}$ if $i=1$

$\left\|f_{a}(x)-A(x)\right\| \leq \frac{\left(\frac{1}{2^{((2 n+1) s-1)}}\right)^{1-1}}{1-\frac{1}{2^{((2 n+1) s-1)}}} \psi(x)=\frac{1}{2^{(2 n+1) s}-2} \frac{\rho}{n}\|x\|^{(2 n+1) s}=\frac{\left.\rho\|x\|\right|^{(2 n+1) s}}{n\left(2^{(2 n+1) s}-2\right)}$.

Finally the inequality (58) holds either, $L=2^{(2 n+1) s-1}$ for $s<\frac{1}{(2 n+1)}$ if $i=0$ and $L=\frac{1}{2^{(2 n+1) s-1}}$ for $s>\frac{1}{(2 n+1)}$ if $i=1$. Now from (59), we prove the following cases for condition (iv).

Case: $1 L=2^{(2 n+1) s-1}$ for $s<\frac{1}{(2 n+1)}$ if $i=0$

$\left\|f_{a}(x)-A(x)\right\| \leq \frac{\left(2^{((2 n+1) s-1)}\right)^{1-0}}{1-2^{((2 n+1) s-1)}} \psi(x)=\frac{1}{2-2^{(2 n+1) s}} \frac{(2 n+2) \rho}{n}\|x\|^{(2 n+1) s}=\frac{(2 n+2) \rho\|x\| \|^{(2 n+1) s}}{n\left(2-2^{(2 n+1) s}\right)}$. 
Case:2 $L=\frac{1}{2^{(2 n+1) s-1}}$ for $s>\frac{1}{(2 n+1)}$ if $i=1$

$\left\|f_{a}(x)-A(x)\right\| \leq \frac{\left(\frac{1}{2^{((2 n+1) s-1)}}\right)^{1-1}}{1-\frac{1}{2^{((2 n+1) s-1)}}} \psi(x)=\frac{1}{2^{(2 n+1) s}-2} \frac{(2 n+2) \rho}{n}\|x\|^{(2 n+1) s}=\frac{\left.(2 n+2) \rho\|x\|\right|^{(2 n+1) s}}{n\left(2^{(2 n+1) s}-2\right)}$.

Hence the proof is complete.

The proof of the following Theorem and corollary is similar tracing to that of Theorem 4.2 and Corollary 4.3. Hence we omit the proofs.

Theorem 4.4 Let $f_{q}: E \rightarrow F$ be a even mapping for which there exist a function $\zeta: E^{2 n+1} \rightarrow[0, \infty)$ with the condition

$\lim _{k \rightarrow \infty} \frac{1}{\kappa_{i}^{2 k}} \zeta\left(\kappa_{i}^{k} x_{0}, \kappa_{i}^{k} x_{1}, \cdots, \kappa_{i}^{k} x_{2 n}, \kappa_{i}^{k} x_{2 n+1}\right)=0$

where $\kappa_{i}$ is defined in (56) such that the functional inequality

$\left\|D f_{q}\left(x_{0}, x_{1}, \cdots, x_{2 n}, x_{2 n+1}\right)\right\| \leq \zeta\left(x_{0}, x_{1}, \cdots, x_{2 n}, x_{2 n+1}\right)$

for all $x_{0}, x_{1}, \cdots, x_{2 n}, x_{2 n+1} \in E$. If there exists $L=L(i)<1$ such that the function

$$
x \rightarrow \psi(x)=\xi\left(\frac{x}{2}\right),
$$

has the property

$\psi(x)=L \kappa_{i}^{2} \psi\left(\kappa_{i} x\right)$.

for all $x \in E$. Then there exists a unique quadratic mapping $Q: E \rightarrow F$ satisfying the functional equation (6) and

$\left\|f_{q}(x)-Q(x)\right\| \leq \frac{L^{1-i}}{1-L} \psi(x)$

for all $x \in E$.

Corollary 4.5 Let $f_{q}: E \rightarrow F$ be a even mapping and there exits real numbers $\rho$ and $s$ such that

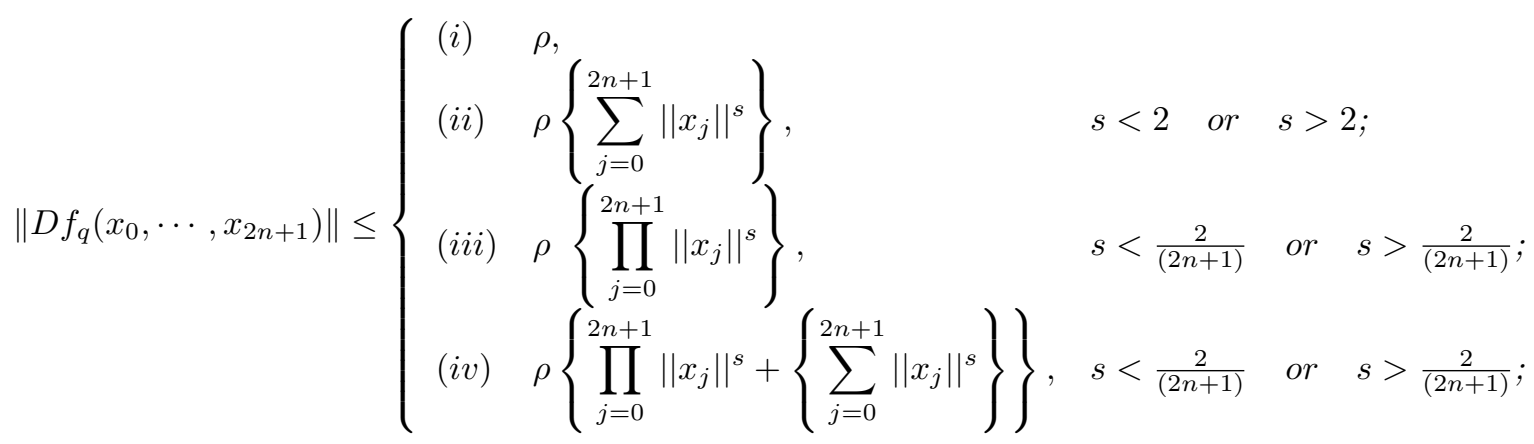

for all $x_{0}, x_{1}, \cdots, x_{2 n}, x_{2 n+1} \in E$. Then there exists a unique quadratic function $Q: E \rightarrow F$ such that

$\left\|f_{q}(x)-Q(x)\right\| \leq \begin{cases}(i) & \frac{2 \rho}{3 n}, \\ \text { (ii) } & \frac{(2 n+1) \rho\|x\|^{s}}{n\left|4-2^{s}\right|}, \\ \text { (iii) } & \frac{\rho \| x||^{(2 n+1) s}}{n\left|4-2^{(2 n+1) s}\right|} \\ \text { (iv) } & \frac{\left.(2 n+2) \rho\|x\|\right|^{(2 n+1) s}}{n\left|4-2^{(2 n+1) s}\right|}\end{cases}$

for all $x \in E$.

Now, we are ready to prove the main fixed point stability results. 
Theorem 4.6 Let $f: E \rightarrow F$ be a mapping for which there exist a function $\zeta: E^{2 n+1} \rightarrow[0, \infty)$ with the conditions (55) and (65) where $\kappa_{i}$ is defined (56) such that the functional inequality

$\left\|D f\left(x_{0}, x_{1}, \cdots, x_{2 n}, x_{2 n+1}\right)\right\| \leq \zeta\left(x_{0}, x_{1}, \cdots, x_{2 n}, x_{2 n+1}\right)$

for all $x_{0}, x_{1}, \cdots, x_{2 n}, x_{2 n+1} \in E$. If there exists $L=L(i)<1$ such that the function

$$
x \rightarrow \psi(x)=\xi\left(\frac{x}{2}\right),
$$

has the properties (58) and (67) for all $x \in E$. Then there exists a unique additive mapping $A: E \rightarrow F$ and a unique quadratic mapping $Q: E \rightarrow F$ satisfying the functional equation (6) and

$\|f(x)-A(x)-Q(x)\| \leq \frac{L^{1-i}}{1-L}(\psi(x)+\psi(-x))$

for all $x \in E$.

Proof. It follows from (48) and Theorem 4.2, we have

$\left\|f_{o}(x)-A(x)\right\| \leq \frac{1}{2} \frac{L^{1-i}}{1-L}(\psi(x)+\psi(-x))$

for all $x \in E$. Also, it follows from (49) and Theorem 4.4, we have

$\left\|f_{e}(x)-Q(x)\right\| \leq \frac{1}{2} \frac{L^{1-i}}{1-L}(\psi(x)+\psi(-x))$

for all $x \in E$. Define

$f(x)=f_{e}(x)+f_{o}(x)$

for all $x \in E$. From (73),(74) and (75), we arrive

$$
\begin{aligned}
\|f(x)-A(x)-Q(x)\|=\left\|f_{e}(x)+f_{o}(x)-A(x)-Q(x)\right\| & \leq\left\|f_{o}(x)-A(x)\right\|+\left\|f_{e}(x)-Q(x)\right\| \\
& \leq \frac{1}{2} \frac{L^{1-i}}{1-L}[(\psi(x)+\psi(-x))+(\psi(x)+\psi(-x))] \\
& \leq \frac{L^{1-i}}{1-L}(\psi(x)+\psi(-x))
\end{aligned}
$$

for all $x \in X$. Hence the theorem is proved.

The following Corollary is an immediate consequence of Theorem 4.6, using Corollaries 4.3 and 4.5 concerning the stability of (6).

Corollary 4.7 Let $f: E \rightarrow F$ be a mapping and there exists real numbers $\rho$ and $s$ such that

$$
\left\|D f\left(x_{0}, \cdots, x_{2 n+1}\right)\right\| \leq \begin{cases}\rho\left\{\sum_{i=0}^{\rho,}\left\|x_{i}\right\|^{s}\right\}, & s \neq 1,2 ; \\ \rho\left\{\prod_{i=0}^{2 n+1}\left\|x_{i}\right\|^{s}\right\}, & s \neq \frac{1}{(2 n+1)}, \frac{2}{(2 n+1)} \\ \rho\left\{\prod_{i=0}^{2 n+1}\left\|x_{i}\right\|^{s}+\left\{\sum_{i=0}^{2 n+1}\left\|x_{i}\right\|^{s}\right\}\right\}, & \neq \frac{1}{(2 n+1)}, \frac{2}{(2 n+1)}\end{cases}
$$

for all $x_{0}, x_{1}, \cdots, x_{2 n}, x_{2 n+1} \in E$. Then there exists a unique additive function $A: E \rightarrow F$ and a unique quadratic function $Q: E \rightarrow F$ such that

$$
\|f(x)-A(x)-Q(x)\| \leq\left\{\begin{array}{l}
\rho\left(\frac{1}{n}+\frac{1}{3 n}\right), \\
(2 n+1) \rho\|x\|^{s}\left(\frac{1}{n\left|2-2^{s}\right|}+\frac{1}{n\left|4-2^{s}\right|}\right), \\
\rho\|x\|^{(2 n+1) s}\left(\frac{1}{n\left|2-2^{(2 n+1) s}\right|}+\frac{1}{n\left|4-2^{(2 n+1) s}\right|}\right), \\
(2 n+2) \rho\|x\|^{(2 n+1) s}\left(\frac{1}{n\left|2-2^{(2 n+1) s}\right|}+\frac{1}{n\left|4-2^{(2 n+1) s}\right|}\right)
\end{array}\right.
$$

for all $x \in E$. 


\section{Basic results in Banach algebra}

Here after, through out this paper, let us consider $X$ and $Y$ to be a normed Algebra and a Banach Algebra, respectively.

Definition 5.1 A C-linear mapping $A: X \rightarrow X$ is called Additive Derivation on $X$ if $A$ satisfies

$A(x y)=A(x) y+x A(y)$

for all $x, y \in X$.

Definition 5.2 A C-linear mapping $A: X \rightarrow X$ is called Generalized Additive Derivation on $X$ if $A$ satisfies

$A\left(x_{1} x_{2} \cdots x_{n}\right)=A\left(x_{1}\right)\left(x_{2} \cdots x_{n}\right)+\cdots \cdots+\left(x_{1} x_{2} \cdots x_{n-1}\right) A\left(x_{n}\right)$

for all $x_{1}, x_{2}, \cdots x_{n} \in X$.

Definition 5.3 A C-linear mapping $Q: X \rightarrow X$ is called Quadratic Derivation on $X$ if $Q$ satisfies

$Q(x y)=Q(x) y^{2}+x^{2} Q(y)$

for all $x, y \in X$.

Definition $5.4 A C$-linear mapping $Q: X \rightarrow X$ is called Generalized Quadratic Derivation on $X$ if $Q$ satisfies

$Q\left(x_{1} x_{2} \cdots x_{n}\right)=Q\left(x_{1}\right)\left(x_{2}^{2} \cdots x_{n}^{2}\right)+\cdots \cdots+\left(x_{1}^{2} x_{2}^{2} \cdots x_{n-1}^{2}\right) Q\left(x_{n}\right)$

for all $x_{1}, x_{2}, \cdots x_{n} \in X$.

\section{Stability results: direct method}

In this section, we investigate the generalized Ulam-Hyers stability of the functional equation (6).

Theorem 6.1 Let $j= \pm 1$. Let $f_{a}: X \rightarrow Y$ be an odd mapping for which there exists a function $\zeta: X^{2 n+1} \rightarrow[0, \infty)$ with the conditions

$\lim _{m \rightarrow \infty} \frac{1}{2^{m j}} \zeta\left(2^{m j} x_{0}, 2^{m j} x_{1}, \cdots, 2^{m j} x_{2 n}, 2^{m j} x_{2 n+1}\right)=0$

$\lim _{m \rightarrow \infty} \frac{1}{2^{(2 n+1) m j}} \rho\left(2^{m j} x_{0}, 2^{m j} x_{1}, \cdots, 2^{m j} x_{2 n}, 2^{m j} x_{2 n+1}\right)=0$

satisfying the functional inequalities

$\left\|D f_{a}\left(x_{0}, x_{1}, \cdots, x_{2 n}, x_{2 n+1}\right)\right\|_{Y} \leq \zeta\left(x_{0}, x_{1}, \cdots, x_{2 n}, x_{2 n+1}\right)$

and

$$
\begin{aligned}
\| f_{a}\left(x_{0} x_{1} \cdots x_{2 n} x_{2 n+1}\right)-f_{a}\left(x_{0}\right)\left(x_{1} \cdots x_{2 n} x_{2 n+1}\right)-\cdots \\
-\left(x_{0} x_{1} \cdots x_{2 n}\right) f_{a}\left(x_{2 n+1}\right) \|_{Y} \leq \rho\left(x_{0}, x_{1}, \cdots, x_{2 n}, x_{2 n+1}\right)
\end{aligned}
$$

for all $x_{0}, x_{1}, \cdots, x_{2 n}, x_{2 n+1} \in X$. Then there exists a unique additive derivation mapping $A: X \rightarrow Y$ satisfying the functional equation (6) and

$\left\|f_{a}(x)-A(x)\right\|_{Y} \leq \frac{1}{2} \sum_{k=\frac{1-j}{2}}^{\infty} \frac{1}{2^{k j}} \xi\left(2^{k j} x\right)$

for all $x \in X$, where $\xi(x)$ and $A(x)$ are defined in (28) and (29) respectively for all $x \in X$. 
Proof. By Theorem 3.1, $A(x)$ is a unique additive mapping which satisfies (6) for all $x_{0}, x_{1}, \cdots, x_{2 n}, x_{2 n+1} \in X$. It follows form (85) and (83),

$$
\begin{aligned}
& \left\|A\left(x_{0} x_{1} \cdots x_{2 n} x_{2 n+1}\right)-A\left(x_{0}\right)\left(x_{1} \cdots x_{2 n} x_{2 n+1}\right)-\cdots-\left(x_{0} x_{1} \cdots x_{2 n}\right) A\left(x_{2 n+1}\right)\right\|_{Y} \\
& =\frac{1}{2^{(2 n+1) m}} \| f_{a}\left(2^{m} x_{0} 2^{m} x_{1} \cdots 2^{m} x_{2 n} 2^{m} x_{2 n+1}\right)-f_{a}\left(2^{m} x_{0}\right)\left(2^{m} x_{1} \cdots 2^{m} x_{2 n} 2^{m} x_{2 n+1}\right)-\cdots \\
& -\left(2^{m} x_{0} 2^{m} x_{1} \cdots 2^{m} x_{2 n}\right) f_{a}\left(2^{m} x_{2 n+1}\right) \|_{Y} \\
& \leq \frac{1}{2^{(2 n+1) m}} \rho\left(2^{m} x_{0}, 2^{m} x_{1}, \cdots, 2^{m} x_{2 n}, 2^{m} x_{2 n+1}\right) \\
& \rightarrow 0 \text { as } m \rightarrow \infty
\end{aligned}
$$

Thus the mapping $A: X \rightarrow Y$ is a unique additive derivation mapping satisfying (6).

Corollary 6.2 Let $\rho$ and $s$ be nonnegative real numbers. Let an odd function $f_{a}: X \rightarrow Y$ satisfies the functional inequalities

$$
\begin{aligned}
& \left\|D f_{a}\left(x_{0}, x_{1}, \cdots, x_{2 n}, x_{2 n+1}\right)\right\|_{Y} \\
& \leq \begin{cases}\rho\left\{\sum_{i=0}^{\rho,}\left\|x_{i}\right\|_{X}^{s}\right\}, & s<1 \quad \text { or } \quad s>1 ; \\
\rho\left\{\prod_{i=0}^{2 n+1}\left\|x_{i}\right\|_{X}^{s}\right\}, & s<\frac{1}{(2 n+1)} \quad \text { or } \quad s>\frac{1}{(2 n+1)} \\
\rho\left\{\prod_{i=0}^{2 n+1}\left\|x_{i}\right\|_{X}^{s}+\left\{\sum_{i=0}^{2 n+1}\left\|x_{i}\right\|_{X}^{s}\right\}\right\}, & s<\frac{1}{(2 n+1)} \quad \text { or } \quad s>\frac{1}{(2 n+1)}\end{cases} \\
& \left\|f_{a}\left(x_{0} x_{1} \cdots x_{2 n} x_{2 n+1}\right)-f_{a}\left(x_{0}\right)\left(x_{1} \cdots x_{2 n} x_{2 n+1}\right)-\cdots-\left(x_{0} x_{1} \cdots x_{2 n}\right) f_{a}\left(x_{2 n+1}\right)\right\|_{Y} \\
& \leq \begin{cases}\rho\left\{\sum_{i=0}^{\rho,}\left\|x_{i}\right\|_{X}^{s}\right\}, & s<1 \quad \text { or } \quad s>1 ; \\
\rho\left\{\prod_{i=0}^{2 n+1}\left\|x_{i}\right\|_{X}^{s}\right\}, & s<\frac{1}{(2 n+1)} \quad \text { or } \quad s>\frac{1}{(2 n+1)} \\
\rho\left\{\prod_{i=0}^{2 n+1}\left\|x_{i}\right\|_{X}^{s}+\left\{\sum_{i=0}^{2 n+1}\left\|x_{i}\right\|_{X}^{s}\right\}\right\}, & s<\frac{1}{(2 n+1)} \quad \text { or } \quad s>\frac{1}{(2 n+1)}\end{cases}
\end{aligned}
$$

for all $x_{0}, x_{1}, \cdots, x_{2 n}, x_{2 n+1} \in X$. Then there exists a unique additive derivation function $A: X \rightarrow Y$ such that

$$
\left\|f_{a}(x)-A(x)\right\|_{Y} \leq\left\{\begin{array}{l}
\frac{\rho}{2 n}, \\
\frac{(2 n+1) \rho\|x\|_{X}^{s}}{2 n\left|2-2^{s}\right|}, \\
\frac{\rho|| x \mid \|_{X}^{(2 n+1) s}}{2 n\left|2-2^{(2 n+1) s}\right|} \\
\frac{(n+1) \rho\|x\|_{X}^{(2 n+1) s}}{n\left|2-2^{(2 n+1) s}\right|}
\end{array}\right.
$$

for all $x \in X$.

Theorem 6.3 Let $j= \pm 1$. Let $f_{q}: X \rightarrow Y$ be an even mapping for which there exists a function $\zeta: X^{2 n+1} \rightarrow[0, \infty)$ with the conditions

$\lim _{m \rightarrow \infty} \frac{1}{4^{m j}} \zeta\left(2^{m j} x_{0}, 2^{m j} x_{1}, \cdots, 2^{m j} x_{2 n}, 2^{m j} x_{2 n+1}\right)=0$ 
$\lim _{m \rightarrow \infty} \frac{1}{4^{(2 n+1) m j}} \rho\left(2^{m j} x_{0}, 2^{m j} x_{1}, \cdots, 2^{m j} x_{2 n}, 2^{m j} x_{2 n+1}\right)=0$

satisfying the functional inequalities

$\left\|D f_{q}\left(x_{0}, x_{1}, \cdots, x_{2 n}, x_{2 n+1}\right)\right\|_{Y} \leq \zeta\left(x_{0}, x_{1}, \cdots, x_{2 n}, x_{2 n+1}\right)$

and

$$
\begin{aligned}
\| f_{q}\left(x_{0} x_{1} \cdots x_{2 n} x_{2 n+1}\right)-f_{q}\left(x_{0}\right)\left(x_{1}^{2} \cdots x_{2 n}^{2} x_{2 n+1}^{2}\right)-\cdots \\
-\left(x_{0}^{2} x_{1}^{2} \cdots x_{2 n}^{2}\right) f_{q}\left(x_{2 n+1}\right) \|_{Y} \leq \rho\left(x_{0}, x_{1}, \cdots, x_{2 n}, x_{2 n+1}\right)
\end{aligned}
$$

for all $x_{0}, x_{1}, \cdots, x_{2 n}, x_{2 n+1} \in X$. Then there exists a unique quadratic derivation mapping $Q: X \rightarrow Y$ satisfying the functional equation (6) and

$\left\|f_{q}(x)-Q(x)\right\|_{Y} \leq \frac{1}{4} \sum_{k=\frac{1-j}{2}}^{\infty} \frac{1}{4^{k j}} \xi\left(2^{k j} x\right)$

for all $x \in X$, where $\xi(x)$ and $Q(x)$ are defined in (28) and (41) respectively for all $x \in X$.

Proof. By Theorem 3.3, $Q(x)$ is a unique quadratic derivation mapping which satisfies (6) for all $x_{0}, x_{1}, \cdots, x_{2 n}, x_{2 n+1} \in$ $X$. It follows form (93) and (91),

$$
\begin{aligned}
& \left\|Q\left(x_{0} x_{1} \cdots x_{2 n} x_{2 n+1}\right)-Q\left(x_{0}\right)\left(x_{1}^{2} \cdots x_{2 n}^{2} x_{2 n+1}^{2}\right)-\cdots-\left(x_{0}^{2} x_{1}^{2} \cdots x_{2 n}^{2}\right) Q\left(x_{2 n+1}\right)\right\|_{Y} \\
& \begin{array}{l}
=\frac{1}{2^{(2 n+1) m}} \| f_{q}\left(2^{m} x_{0} 2^{m} x_{1} \cdots 2^{m} x_{2 n} 2^{m} x_{2 n+1}\right)-f_{q}\left(2^{m} x_{0}\right)\left(2^{m} x_{1}^{2} \cdots 2^{m} x_{2 n}^{2} 2^{m} x_{2 n+1}^{2}\right)-\cdots \\
\quad-\left(2^{m} x_{0}^{2} 2^{m} x_{1}^{2} \cdots 2^{m} x_{2 n}^{2}\right) f_{q}\left(2^{m} x_{2 n+1}\right) \|_{Y} \\
\leq \frac{1}{2^{(2 n+1) m}} \rho\left(2^{m} x_{0}, 2^{m} x_{1}, \cdots, 2^{m} x_{2 n}, 2^{m} x_{2 n+1}\right) \\
\rightarrow 0 \quad \text { as } m \rightarrow \infty
\end{array}
\end{aligned}
$$

Thus the mapping $Q: X \rightarrow Y$ is a unique quadratic derivation mapping satisfying (6).

Corollary 6.4 Let $\rho$ and $s$ be nonnegative real numbers. Let an even function $f_{q}: X \rightarrow Y$ satisfies the inequality

$$
\begin{aligned}
& \left\|D f_{q}\left(x_{0}, x_{1}, \cdots, x_{2 n}, x_{2 n+1}\right)\right\|_{Y} \\
& \leq \begin{cases}\rho\left\{\sum_{i=0}^{\rho,}\left\|x_{i}\right\|_{X}^{s}\right\}, & s<2 \quad \text { or } \quad s>2 ; \\
\rho\left\{\prod_{i=0}^{2 n+1}\left\|x_{i}\right\|_{X}^{s}\right\}, & s<\frac{2}{(2 n+1)} \quad \text { or } \quad s>\frac{2}{(2 n+1)} \\
\rho\left\{\prod_{i=0}^{2 n+1}\left\|x_{i}\right\|_{X}^{s}+\left\{\sum_{i=0}^{2 n+1}\left\|x_{i}\right\|_{X}^{s}\right\}\right\}, & s<\frac{2}{(2 n+1)} \quad \text { or } \quad s>\frac{2}{(2 n+1)}\end{cases} \\
& \left\|f_{q}\left(x_{0} x_{1} \cdots x_{2 n} x_{2 n+1}\right)-f_{q}\left(x_{0}\right)\left(x_{1}^{2} \cdots x_{2 n}^{2} x_{2 n+1}^{2}\right)-\cdots-\left(x_{0}^{2} x_{1}^{2} \cdots x_{2 n}^{2}\right) f_{q}\left(x_{2 n+1}\right)\right\|_{Y} \\
& \leq \begin{cases}\rho\left\{\sum_{i=0}^{\rho n}\left\|x_{i}\right\|_{X}^{s}\right\}, & s<2 \quad \text { or } \quad s>2 ; \\
\rho\left\{\prod_{i=0}^{2 n+1}\left\|x_{i}\right\|_{X}^{s}\right\}, & s<\frac{2}{(2 n+1)} \quad \text { or } \quad s>\frac{2}{(2 n+1)} \\
\rho\left\{\prod_{i=0}^{2 n+1}\left\|x_{i}\right\|_{X}^{s}+\left\{\sum_{i=0}^{2 n+1}\left\|x_{i}\right\|_{X}^{s}\right\}\right\}, & s<\frac{2}{(2 n+1)} \quad \text { or } \quad s>\frac{2}{(2 n+1)}\end{cases}
\end{aligned}
$$


for all $x_{0}, x_{1}, \cdots, x_{2 n}, x_{2 n+1} \in X$. Then there exists a unique quadratic derivation function $Q: X \rightarrow Y$ such that

$$
\left\|f_{q}(x)-Q(x)\right\|_{Y} \leq\left\{\begin{array}{l}
\frac{\rho}{6 n}, \\
\frac{(2 n+1) \rho\|x\|_{X}^{s}}{2 n\left|4-2^{s}\right|} \\
\frac{\rho|| x \|_{X}^{(2 n+1) s}}{2 n\left|4-2^{(2 n+1) s}\right|} \\
\frac{(n+1) \rho\|x\|_{X}^{(2 n+1) s}}{n\left|4-2^{(2 n+1) s}\right|}
\end{array}\right.
$$

for all $x \in X$.

Theorem 6.5 Let $j= \pm 1$. Let $f: X \rightarrow Y$ be a mapping for which there exist a function $\zeta: X^{2 n+1} \rightarrow[0, \infty)$ with the conditions (82), (83), (90)and (91) such that the functional inequalities

$\left\|D f\left(x_{0}, x_{1}, \cdots, x_{2 n}, x_{2 n+1}\right)\right\|_{Y} \leq \zeta\left(x_{0}, x_{1}, \cdots, x_{2 n}, x_{2 n+1}\right)$

and (84), (85), (92)and (93) for all $x_{0}, x_{1}, \cdots, x_{2 n}, x_{2 n+1} \in X$. Then there exists a unique additive derivation mapping $A: X \rightarrow Y$ and a unique quadratic derivation mapping $Q: X \rightarrow Y$ such that

$\|f(x)-A(x)-Q(x)\|_{Y} \leq \frac{1}{2}\left[\frac{1}{2} \sum_{k=\frac{1-j}{2}}^{\infty}\left(\frac{\xi\left(2^{k j} x\right)}{2^{k j}}+\frac{\xi\left(-2^{k j} x\right)}{2^{k j}}\right)+\frac{1}{4} \sum_{k=\frac{1-j}{2}}^{\infty}\left(\frac{\xi\left(2^{k j} x\right)}{4^{k j}}+\frac{\xi\left(-2^{k j} x\right)}{4^{k j}}\right)\right]$

for all $x \in X$, where $\xi(x), A(x)$ and $Q(x)$ are defined in (28), (29) and (41) respectively for all $x \in X$.

Proof. The proof is similar to that of Theorem 3.5.

Corollary 6.6 Let $\rho$ and $s$ be nonnegative real numbers. Let a function $f: X \rightarrow Y$ satisfies the inequality

$$
\begin{aligned}
& \left\|D f\left(x_{0}, x_{1}, \cdots, x_{2 n}, x_{2 n+1}\right)\right\|_{Y} \\
& \leq \begin{cases}\rho\left\{\sum_{i=0}^{\rho,}\left\|x_{i}\right\|_{X}^{s}\right\}, & s \neq 1,2 ; \\
\rho\left\{\prod_{i=0}^{2 n+1}\left\|x_{i}\right\|_{X}^{s}\right\}, & s \neq \frac{1}{(2 n+1)}, \frac{2}{(2 n+1)} ; \\
\rho\left\{\prod_{i=0}^{2 n+1}\left\|x_{i}\right\|_{X}^{s}+\left\{\sum_{i=0}^{2 n+1}\left\|x_{i}\right\|_{X}^{s}\right\}\right\}, & s \neq \frac{1}{(2 n+1)}, \frac{2}{(2 n+1)} ;\end{cases}
\end{aligned}
$$

for all $x_{0}, x_{1}, \cdots, x_{2 n}, x_{2 n+1} \in X$. Then there exists a unique additive derivation function $A: X \rightarrow Y$ and a unique quadratic derivation function $Q: X \rightarrow Y$ such that

$$
\|f(x)-A(x)-Q(x)\|_{Y} \leq\left\{\begin{array}{l}
\rho\left(\frac{1}{2 n}+\frac{1}{6 n}\right), \\
(2 n+1) \rho|| x \|^{s}\left(\frac{1}{2 n\left|2-2^{s}\right|}+\frac{1}{2 n\left|4-2^{s}\right|}\right), \\
\rho\|x\|^{(2 n+1) s}\left(\frac{1}{2 n\left|2-2^{(2 n+1) s}\right|}+\frac{1}{2 n\left|4-2^{(2 n+1) s}\right|}\right), \\
(2 n+2) \rho\|x\|^{(2 n+1) s}\left(\frac{1}{n\left|2-2^{(2 n+1) s}\right|}+\frac{1}{n\left|4-2^{(2 n+1) s}\right|}\right)
\end{array}\right.
$$

for all $x \in X$.

\section{Stability results: fixed point method}

Theorem 7.1 Let $f_{a}: X \rightarrow Y$ be a odd mapping for which there exist a function $\zeta: X^{2 n+1} \rightarrow[0, \infty)$ with the conditions

$\lim _{k \rightarrow \infty} \frac{1}{\kappa_{i}^{k}} \zeta\left(\kappa_{i}^{k} x_{0}, \kappa_{i}^{k} x_{1}, \cdots, \kappa_{i}^{k} x_{2 n}, \kappa_{i}^{k} x_{2 n+1}\right)=0$ 
$\lim _{k \rightarrow \infty} \frac{1}{\kappa_{i}^{(2 n+1) k}} \rho\left(\kappa_{i}^{k} x_{0}, \kappa_{i}^{k} x_{1}, \cdots, \kappa_{i}^{k} x_{2 n}, \kappa_{i}^{k} x_{2 n+1}\right)=0$

where $\kappa_{i}$ is defined in (56) satisfying the functional inequalities

$\left\|D f_{a}\left(x_{0}, x_{1}, \cdots, x_{2 n}, x_{2 n+1}\right)\right\|_{Y} \leq \zeta\left(x_{0}, x_{1}, \cdots, x_{2 n}, x_{2 n+1}\right)$

and

$$
\begin{aligned}
\| f_{a}\left(x_{0} x_{1} \cdots x_{2 n} x_{2 n+1}\right)-f_{a}\left(x_{0}\right)\left(x_{1} \cdots x_{2 n} x_{2 n+1}\right)-\cdots \\
-\left(x_{0} x_{1} \cdots x_{2 n}\right) f_{a}\left(x_{2 n+1}\right) \|_{Y} \leq \rho\left(x_{0}, x_{1}, \cdots, x_{2 n}, x_{2 n+1}\right)
\end{aligned}
$$

for all $x_{0}, x_{1}, \cdots, x_{2 n}, x_{2 n+1} \in X$. If there exists $L=L(i)<1$ such that the function

$$
x \rightarrow \psi(x)=\xi\left(\frac{x}{2}\right),
$$

has the property

$\psi(x)=L \kappa_{i} \psi\left(\kappa_{i} x\right)$

for all $s \in X$. Then there exists a unique additive derivation mapping $A: X \rightarrow Y$ satisfying the functional equation (6) and

$\left\|f_{a}(x)-A(x)\right\|_{Y} \leq \frac{L^{1-i}}{1-L} \psi(x)$

for all $x \in X$.

Proof. By Theorem 4.2, $A(x)$ is a unique additive derivation mapping which satisfies (6) for all $x \in X$. It follows form (105) and (103),

$$
\begin{aligned}
& \left\|A\left(x_{0} x_{1} \cdots x_{2 n} x_{2 n+1}\right)-A\left(x_{0}\right)\left(x_{1} \cdots x_{2 n} x_{2 n+1}\right)-\cdots-\left(x_{0} x_{1} \cdots x_{2 n}\right) A\left(x_{2 n+1}\right)\right\|_{Y} \\
& =\frac{1}{\kappa_{i}^{(2 n+1) m}} \| f\left(\kappa_{i}^{k} x_{0} \kappa_{i}^{k} x_{1} \cdots \kappa_{i}^{k} x_{2 n} \kappa_{i}^{k} x_{2 n+1}\right)-f\left(\kappa_{i}^{k} x_{0}\right)\left(\kappa_{i}^{k} x_{1} \cdots \kappa_{i}^{k} x_{2 n} \kappa_{i}^{k} x_{2 n+1}\right)-\cdots \\
& \quad-\left(\kappa_{i}^{k} x_{0} \kappa_{i}^{k} x_{1} \cdots \kappa_{i}^{k} x_{2 n}\right) f\left(\kappa_{i}^{k} x_{2 n+1}\right) \|_{Y} \\
& \leq \frac{1}{\kappa_{i}^{(2 n+1) m}} \rho\left(2^{m} x_{0}, 2^{m} x_{1}, \cdots, 2^{m} x_{2 n}, 2^{m} x_{2 n+1}\right) \\
& \rightarrow 0 \text { as } m \rightarrow \infty
\end{aligned}
$$

Thus the mapping $A: X \rightarrow Y$ is a unique additive derivation mapping satisfying (6).

Corollary 7.2 Let $f_{a}: X \rightarrow Y$ be a odd mapping and there exists real numbers $\rho$ and $s$ such that

$$
\begin{aligned}
& \left\|D f_{a}\left(x_{0}, x_{1}, \cdots, x_{2 n}, x_{2 n+1}\right)\right\|_{Y}
\end{aligned}
$$

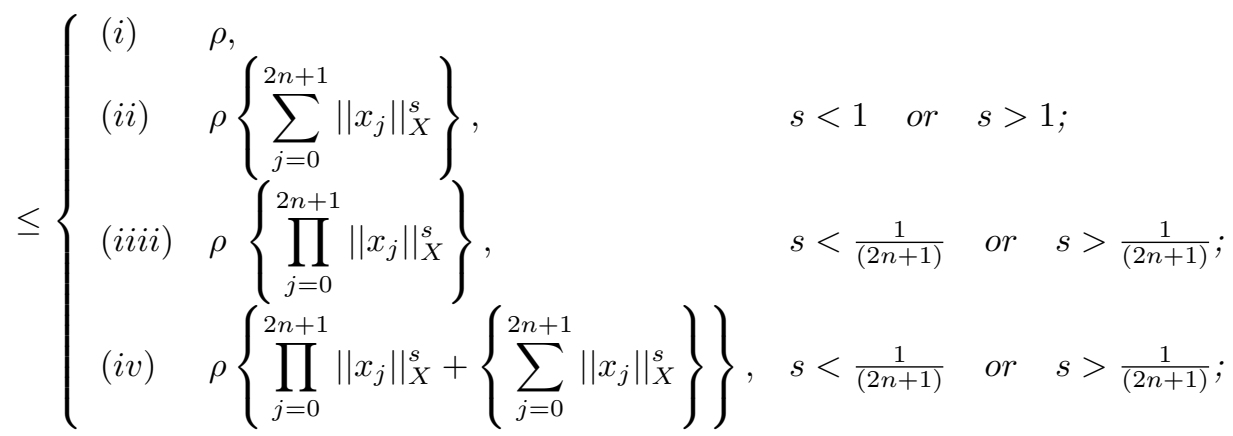




$$
\begin{aligned}
& \left\|f_{a}\left(x_{0} x_{1} \cdots x_{2 n} x_{2 n+1}\right)-f_{a}\left(x_{0}\right)\left(x_{1} \cdots x_{2 n} x_{2 n+1}\right)-\cdots-\left(x_{0} x_{1} \cdots x_{2 n}\right) f_{a}\left(x_{2 n+1}\right)\right\|_{Y}
\end{aligned}
$$

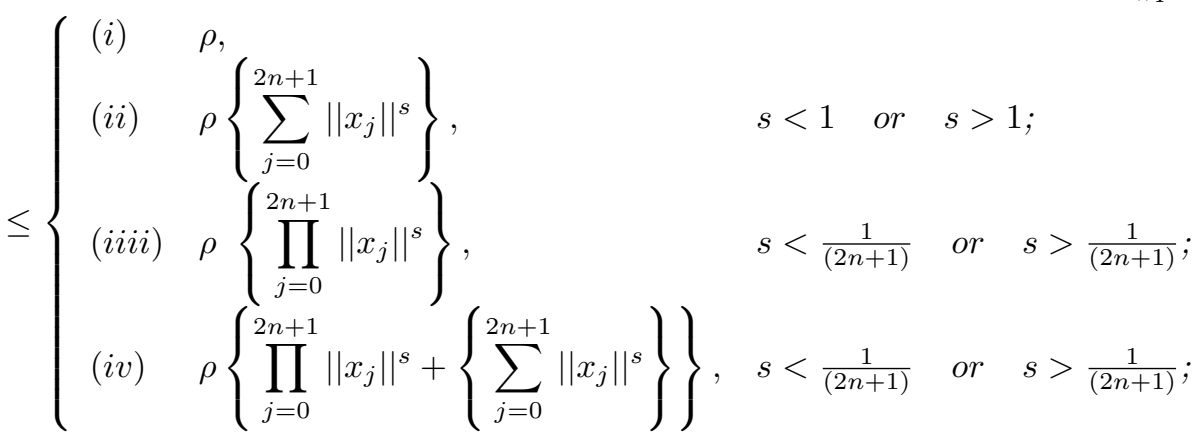

for all $x_{0}, x_{1}, \cdots, x_{2 n}, x_{2 n+1} \in X$. Then there exists a unique additive derivation $A: X \rightarrow Y$ such that

$$
\left\|f_{a}(x)-A(x)\right\|_{Y} \leq \begin{cases}(i) & \frac{\rho}{2 n}, \\ \text { (ii) } & \frac{\left.(2 n+1) \rho\|x\|\right|^{s}}{n\left|2-2^{s}\right|}, \\ \text { (iii) } & \frac{\rho|| x||^{(2 n+1) s}}{n \mid 2-2^{(2 n+1) s \mid}} \\ \text { (iv) } & \frac{\left.(2 n+2) \rho\|x\|\right|^{(2 n+1) s}}{n\left|2-2^{(2 n+1) s}\right|}\end{cases}
$$

for all $x \in X$.

Theorem 7.3 Let $f_{q}: X \rightarrow Y$ be a even mapping for which there exists a function $\zeta: X^{2 n+1} \rightarrow[0, \infty)$ with the conditions

$\lim _{k \rightarrow \infty} \frac{1}{\kappa_{i}^{2 k}} \zeta\left(\kappa_{i}^{k} x_{0}, \kappa_{i}^{k} x_{1}, \cdots, \kappa_{i}^{k} x_{2 n}, \kappa_{i}^{k} x_{2 n+1}\right)=0$

$\lim _{k \rightarrow \infty} \frac{1}{\kappa_{i}^{2 k}} \rho\left(\kappa_{i}^{k} x_{0}, \kappa_{i}^{k} x_{1}, \cdots, \kappa_{i}^{k} x_{2 n}, \kappa_{i}^{k} x_{2 n+1}\right)=0$

where $\kappa_{i}$ is defined in (56) satisfying the functional inequalities

$\left\|D f_{q}\left(x_{0}, x_{1}, \cdots, x_{2 n}, x_{2 n+1}\right)\right\|_{Y} \leq \zeta\left(x_{0}, x_{1}, \cdots, x_{2 n}, x_{2 n+1}\right)$

and

$$
\begin{aligned}
\| f_{q}\left(x_{0} x_{1} \cdots x_{2 n} x_{2 n+1}\right)-f_{q}\left(x_{0}\right)\left(x_{1}^{2} \cdots x_{2 n}^{2} x_{2 n+1}^{2}\right)-\cdots \\
-\left(x_{0}^{2} x_{1}^{2} \cdots x_{2 n}^{2}\right) f_{q}\left(x_{2 n+1}\right) \|_{Y} \leq \rho\left(x_{0}, x_{1}, \cdots, x_{2 n}, x_{2 n+1}\right)
\end{aligned}
$$

for all $x_{0}, x_{1}, \cdots, x_{2 n}, x_{2 n+1} \in X$. If there exists $L=L(i)<1$ such that the function

$$
x \rightarrow \psi(x)=\xi\left(\frac{x}{2}\right),
$$

has the property

$\psi(x)=L \kappa_{i}^{2} \psi\left(\kappa_{i} x\right)$.

for all $x \in X$. Then there exists a unique quadratic derivation mapping $Q: X \rightarrow Y$ satisfying the functional equation (6) and

$\left\|f_{q}(x)-Q(x)\right\|_{Y} \leq \frac{L^{1-i}}{1-L} \psi(x)$

for all $x \in X$. 
Proof. By Theorem 4.4, $Q(x)$ is a unique quadratic derivation mapping which satisfies (6) for all $x \in X$. It follows form (114) and (112),

$$
\begin{aligned}
& \left\|Q\left(x_{0} x_{1} \cdots x_{2 n} x_{2 n+1}\right)-Q\left(x_{0}\right)\left(x_{1}^{2} \cdots x_{2 n}^{2} x_{2 n+1}^{2}\right)-\cdots-\left(x_{0}^{2} x_{1}^{2} \cdots x_{2 n}^{2}\right) Q\left(x_{2 n+1}\right)\right\|_{Y} \\
& =\frac{1}{\kappa_{i}^{(2 n+1) m}} \| f_{q}\left(\kappa_{i}^{2 k} x_{0} \kappa_{i}^{2 k} x_{1} \cdots \kappa_{i}^{2 k} x_{2 n} \kappa_{i}^{2 k} x_{2 n+1}\right)-f_{q}\left(\kappa_{i}^{2 k} x_{0}\right)\left(\kappa_{i}^{2 k} x_{1}^{2} \cdots \kappa_{i}^{2 k} x_{2 n}^{2} \kappa_{i}^{2 k} x_{2 n+1}^{2}\right)-\cdots \\
& \quad \quad-\left(\kappa_{i}^{2 k} x_{0}^{2} \kappa_{i}^{2 k} x_{1}^{2} \cdots \kappa_{i}^{2 k} x_{2 n}^{2}\right) f_{q}\left(\kappa_{i}^{2 k} x_{2 n+1}\right) \|_{Y} \\
& \leq \frac{1}{\kappa_{i}^{(2 n+1) m}} \rho\left(\kappa_{i}^{2 k} x_{0}, \kappa_{i}^{2 k} x_{1}, \cdots, \kappa_{i}^{2 k} x_{2 n}, \kappa_{i}^{2 k} x_{2 n+1}\right) \\
& \rightarrow 0 \quad \text { as } m \rightarrow \infty
\end{aligned}
$$

Thus the mapping $Q: X \rightarrow Y$ is a unique quadratic derivation mapping satisfying (6).

Corollary 7.4 Let $f_{q}: X \rightarrow Y$ be a even mapping and there exists real numbers $\rho$ and s such that

$\left\|D f_{q}\left(x_{0}, x_{1}, \cdots, x_{2 n}, x_{2 n+1}\right)\right\|_{Y}$

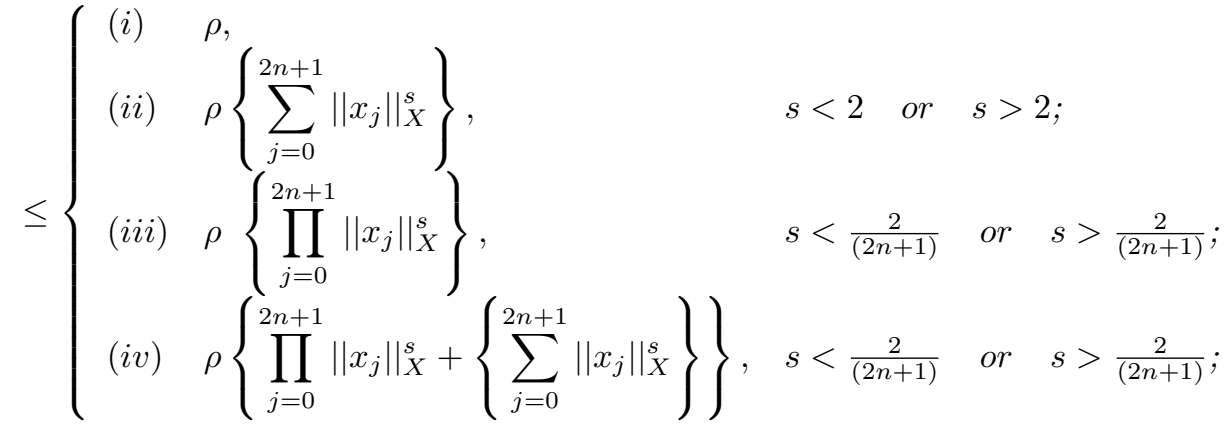

$$
\left\|f_{q}\left(x_{0} x_{1} \cdots x_{2 n} x_{2 n+1}\right)-f_{q}\left(x_{0}\right)\left(x_{1}^{2} \cdots x_{2 n}^{2} x_{2 n+1}^{2}\right)-\cdots-\left(x_{0}^{2} x_{1}^{2} \cdots x_{2 n}^{2}\right) f_{q}\left(x_{2 n+1}\right)\right\|_{Y}
$$

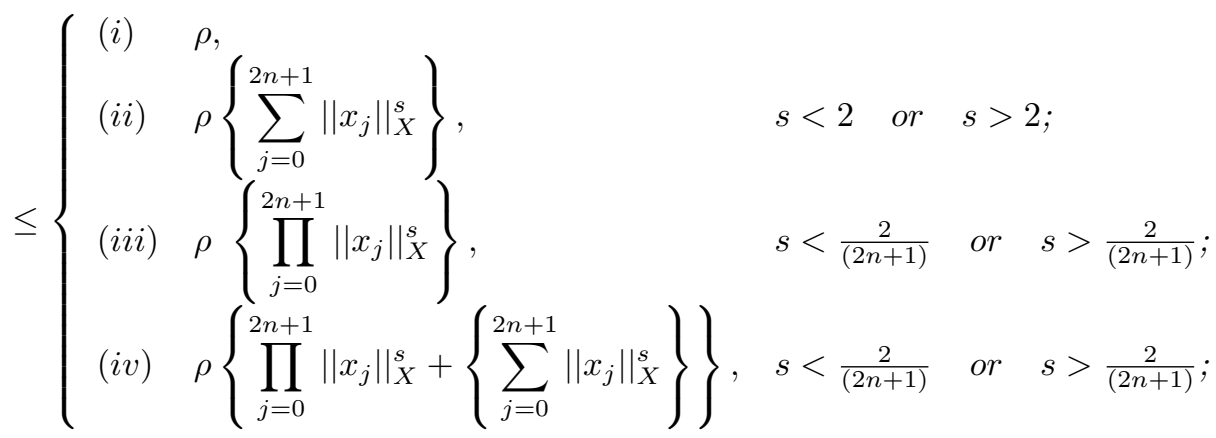

for all $x_{0}, x_{1}, \cdots, x_{2 n}, x_{2 n+1} \in X$. Then there exists a unique quadratic derivation $Q: X \rightarrow Y$ such that

$$
\left\|f_{q}(x)-Q(x)\right\|_{Y} \leq \begin{cases}\text { (i) } & \frac{\rho}{6 n}, \\ \text { (ii) } & \frac{(2 n+1) \rho\|x\|^{s}}{n\left|4-2^{s}\right|}, \\ \text { (iii) } & \frac{\left.\rho\|x\|\right|^{(2 n+1) s}}{n \mid 4-2^{(2 n+1) s \mid}} \\ \text { (iv) } & \frac{\left.(n+1) \rho\|x\|\right|^{(2 n+1) s}}{n\left|4-2^{(2 n+1) s}\right|}\end{cases}
$$

for all $x \in X$.

Theorem 7.5 Let $f: X \rightarrow Y$ be a mapping for which there exist a function $\zeta: X^{2 n+1} \rightarrow[0, \infty)$ with the conditions (102), (103), (111) and (112) where $\kappa_{i}$ is defined in (56) such that the functional inequality

$\left\|D f\left(x_{0}, x_{1}, \cdots, x_{2 n}, x_{2 n+1}\right)\right\|_{Y} \leq \zeta\left(x_{0}, x_{1}, \cdots, x_{2 n}, x_{2 n+1}\right)$ 
and (105), (114) for all $x_{0}, x_{1}, \cdots, x_{2 n}, x_{2 n+1} \in X$. If there exists $L=L(i)<1$ such that the function

$$
x \rightarrow \psi(x)=\xi\left(\frac{x}{2}\right),
$$

has the properties (106) and (115) for all $x \in X$. Then there exists a unique additive derivation mapping $A: X \rightarrow Y$ and a unique quadratic derivation mapping $Q: X \rightarrow Y$ satisfying the functional equation (6) and

$\|f(x)-A(x)-Q(x)\|_{Y} \leq \frac{L^{1-i}}{1-L}(\psi(x)+\psi(-x))$

for all $x \in X$.

Corollary 7.6 Let $f: X \rightarrow Y$ be a mapping and there exists real numbers $\rho$ and $s$ such that

$\left\|D f\left(x_{0}, x_{1}, \cdots, x_{2 n}, x_{2 n+1}\right)\right\|_{Y}$

$$
\leq \begin{cases}\rho\left\{\sum_{i=0}^{\rho}\left\|x_{i}\right\|^{s}\right\}, & s \neq 1,2 \\ \rho\left\{\prod_{i=0}^{2 n+1}\left\|x_{i}\right\|^{s}\right\}, & s \neq \frac{1}{(2 n+1)}, \frac{2}{(2 n+1)} \\ \rho\left\{\prod_{i=0}^{2 n+1}\left\|x_{i}\right\|^{s}+\left\{\sum_{i=0}^{2 n+1}\left\|x_{i}\right\|^{s}\right\}\right\}, & \neq \frac{1}{(2 n+1)}, \frac{2}{(2 n+1)}\end{cases}
$$

and (105), (114) for all $x_{0}, x_{1}, \cdots, x_{2 n}, x_{2 n+1} \in X$. Then there exists a unique additive derivation function $A$ : $X \rightarrow Y$ and a unique quadratic derivation function $Q: X \rightarrow Y$ such that

$$
\begin{aligned}
& \|f(x)-A(x)-Q(x)\|_{Y} \\
& \leq\left\{\begin{array}{l}
\rho\left(\frac{1}{2 n}+\frac{1}{6 n}\right), \\
(2 n+1) \rho\|x\|^{s}\left(\frac{1}{n\left|2-2^{s}\right|}+\frac{1}{n\left|4-2^{s}\right|}\right), \\
\rho\|x\|^{(2 n+1) s}\left(\frac{1}{\left.n \mid 2-2^{(2 n+1) s \mid}+\frac{1}{n\left|4-2^{(2 n+1) s}\right|}\right),}\right. \\
(2 n+2) \rho\|x\|^{(2 n+1) s}\left(\frac{1}{n \mid 2-2^{(2 n+1) s \mid}}+\frac{1}{n \mid 4-2^{(2 n+1) s \mid}}\right)
\end{array}\right.
\end{aligned}
$$

for all $x \in X$.

\section{Acknowledgements}

This work is dedicated to all Mathematicians and Research Scholars those who are working in functional equations.

\section{References}

[1] J. Aczel and J. Dhombres, Functional Equations in Several Variables, Cambridge Univ, Press, 1989.

[2] T. Aoki, On the stability of the linear transformation in Banach spaces, J. Math. Soc. Japan, 2 (1950), 64-66.

[3] M. Arunkumar, S. Karthikeyan, Solution and stability of $n$-dimensional mixed Type additive and quadratic functional equation, Far East Journal of Applied Mathematics, Volume 54, Number 1, 2011, 47-64.

[4] M. Arunkumar, John M. Rassias, On the generalized Ulam-Hyers stability of an AQ-mixed type functional equation with counter examples, Far East Journal of Applied Mathematics, Volume 71, No. 2, (2012), 279-305.

[5] C.Borelli, G.L.Forti, On a general Hyers-Ulam stability, Internat J.Math.Math.Sci, 18 (1995), 229-236.

[6] I.S. Chang, E.H. Lee, H.M. Kim, On the Hyers-Ulam-Rassias stability of a quadratic functional equations, Math. Ineq. Appl., 6(1) (2003), 87-95. 
[7] P.W.Cholewa, Remarks on the stability of functional equations , Aequationes Math., 27 (1984), 76-86.

[8] S.Czerwik, On the stability of the quadratic mappings in normed spaces,Abh.Math.Sem.Univ Hamburg., 62 (1992), 59-64.

[9] S. Czerwik, Functional Equations and Inequalities in Several Variables, World Scientific, River Edge, NJ, 2002.

[10] P. Gavruta, A generalization of the Hyers-Ulam-Rassias stability of approximately additive mappings , J. Math. Anal. Appl., 184 (1994), 431-436.

[11] M. Eshaghi Gordji, H. Khodaie, Solution and stability of generalized mixed type cubic, quadratic and additive functional equation in quasi-Banach spaces, arxiv: 0812. 2939v1 Math FA, 15 Dec 2008.

[12] M. Eshaghi Gordji, H. Khodaei, J.M. Rassias, Fixed point methods for the stability of general quadratic functional equation, Fixed Point Theory, 12 (2011), no. 1, 71-82.

[13] D.H. Hyers, On the stability of the linear functional equation, Proc.Nat. Acad.Sci.,U.S.A., 27 (1941) $222-224$.

[14] D.H. Hyers, G. Isac, Th.M. Rassias, Stability of functional equations in several variables, Birkhauser, Basel, 1998.

[15] K.W. Jun, H.M. Kim, On the stability of an n-dimensional quadratic and additive type functional equation, Math. Ineq. Appl, 9(1) (2006), 153-165.

[16] S.M. Jung, On the Hyers-Ulam stability of the functional equations that have the quadratic property, J. Math. Anal. Appl. 222 (1998), 126-137.

[17] S.M. Jung, Hyers-Ulam-Rassias Stability of Functional Equations in Mathematical Analysis, Hadronic Press, Palm Harbor, 2001.

[18] Pl. Kannappan, Quadratic functional equation inner product spaces, Results Math., 27, No.3-4, (1995), 368-372.

[19] Pl. Kannappan, Functional Equations and Inequalities with Applications, Springer Monographs in Mathematics, 2009.

[20] B.Margoils, J.B.Diaz, A fixed point theorem of the alternative for contractions on a generalized complete metric space, Bull.Amer. Math. Soc., 12674 (1968), 305-309.

[21] J.M. Rassias, On approximately of approximately linear mappings by linear mappings, J. Funct. Anal. USA, 46, (1982) 126-130.

[22] J.M. Rassias, H.M. Kim, Generalized Hyers-Ulam stability for general additive functional equations in quasi- $\beta$-normed spaces, J. Math. Anal. Appl., 356 (2009), no. 1, 302-309.

[23] Th.M. Rassias, On the stability of the linear mapping in Banach spaces, Proc.Amer.Math. Soc., 72 (1978), 297-300.

[24] Matina J. Rassias, M. Arunkumar, S. Ramamoorthi, Stability of the Leibniz additive-quadratic functional equation in Quasi-Beta normed space: Direct and fixed point methods, Journal Of Concrete And Applicable Mathematics (JCAAM), Vol. 14 No. 1-2, (2014), 22 - 46.

[25] Th.M. Rassias, Functional Equations, Inequalities and Applications, Kluwer Acedamic Publishers, Dordrecht, Bostan London, 2003.

[26] K. Ravi, M. Arunkumar and J.M. Rassias, On the Ulam stability for the orthogonally general Euler-Lagrange type functional equation, International Journal of Mathematical Sciences, Autumn 2008 Vol.3, No. 08, 36-47.

[27] F.Skof, Proprietà locali e approssimazione di operatori,Rend. Sem. Mat.Fis. Milano, 53 (1983), 113-129.

[28] S.M. Ulam, Problems in Modern Mathematics, Science Editions, Wiley, NewYork, 1964.

[29] T.Z. Xu, J.M. Rassias, M.J. Rassias, W.X. Xu, A fixed point approach to the stability of quintic and sextic functional equations in quasi- $\beta$-normed spaces, J. Inequal. Appl., 2010, Art. ID 423231, 23 pp. 